Discussion Paper No. 15-070

Steuerpolitik in Deutschland eine Halbzeitbilanz der aktuellen

Legislaturperiode im Kontext europäischer Entwicklungen

Rainer Bräutigam and Christoph Spengel

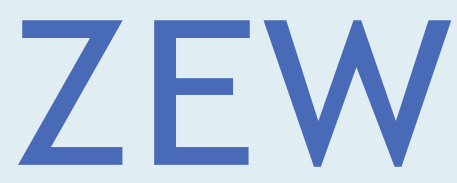

Zentrum für Europäische Wirtschaftsforschung $\mathrm{GmbH}$ Centre for European Economic Research 
Discussion Paper No. 15-070

\title{
Steuerpolitik in Deutschland - eine Halbzeitbilanz der aktuellen Legislaturperiode im Kontext europäischer Entwicklungen
}

\author{
Rainer Bräutigam and Christoph Spengel
}

Download this ZEW Discussion Paper from our ftp server:

http://ftp.zew.de/pub/zew-docs/dp/dp15070.pdf

Die Discussion Papers dienen einer möglichst schnellen Verbreitung von neueren Forschungsarbeiten des ZEW. Die Beiträge liegen in alleiniger Verantwortung der Autoren und stellen nicht notwendigerweise die Meinung des ZEW dar.

Discussion Papers are intended to make results of ZEW research promptly available to other economists in order to encourage discussion and suggestions for revisions. The authors are solely responsible for the contents which do not necessarily represent the opinion of the ZEW. 


\title{
Steuerpolitik in Deutschland - eine Halbzeitbilanz der aktuellen Legislaturperiode im Kontext europäischer Entwicklungen
}

\author{
Rainer Bräutigam (ZEW und Universität Mannheim) \\ Christoph Spengel (Universität Mannheim und ZEW)
}

\section{Zusammenfassung:}

Seit der Unternehmensteuerreform 2008/2009 haben sich die steuerlichen Rahmenbedingungen in Deutschland im Grundsatz nicht geändert. Eine Analyse der deutschen Steuerpolitik in der laufenden Legislaturperiode zeigt, dass sich Änderungen lediglich auf die Beseitigung verfassungswidriger Regelungen beschränken. Aktuell geht es um die Erbschaftsteuer, auch die Grundsteuer ist im Visier des Bundesverfassungsgerichts. Gleichzeitig steigen die Tarife bei den lokalen Steuern. Bei den Ertragsteuern für Kapitalgesellschaften steht diese Entwicklung im Kontrast zu den Entwicklungen in den anderen EU-Mitgliedstaaten, wo die tariflichen Steuersätze immer weiter sinken. Eine quantitative Analyse der effektiven Steuerbelastungen innerhalb der EU zeigt erste Konsequenzen des steuerlichen Stillstands in Deutschland auf. Im Vergleich zu anderen europäischen Ländern werden die steuerlichen Standortbedingungen für Unternehmen in Deutschland zusehends unattraktiver.

JEL Klassifizierung: H24, H25, H71, K34

Stichworte: Steuerpolitik, Deutschland, Europäische Union, Steuerbelastungen, Unternehmensbesteuerung, European Tax Analyzer

Danksagung: Dieser Beitrag ist im Rahmen des MannheimTaxation (MaTax) Science Campus entstanden. Die Autoren bedanken sich für die finanzielle Unterstützung der Leibniz Gemeinschaft, des Landes BadenWürttemberg sowie der teilnehmenden Einrichtungen ZEW und Universität Mannheim. 


\section{Problemstellung, Zielsetzung und Aufbau}

Seit der Unternehmensteuerreform 2008/2009, in dessen Rahmen u.a. der Körperschafsteuersatz von $25 \%$ auf $15 \%$ gesenkt wurde, haben sich die steuerlichen Rahmenbedingungen in Deutschland im Grundsatz nicht geändert. Zur Halbzeit der derzeitigen Legislaturperiode wird deshalb eine Zwischenbilanz der Steuerpolitik in Deutschland gezogen. Dazu wird zunächst auf Entwicklungen im Rahmen der Körperschaft- und Einkommensteuer, der Realsteuern (Gewerbe-/Grundsteuer) sowie der Erbschaftsteuer in Deutschland eingegangen. Daneben werden EU-weite Entwicklungen erfasst, um Aussagen hinsichtlich der Position Deutschlands innerhalb des EU-weiten Steuerwettbewerbs zu treffen. Dazu wird auf die wichtigsten Änderungen im Zeitraum 2013 bis 2015 bei der Besteuerung von Kapitalgesellschaften und ihrer Anteilseigner in den 28 EU-Mitgliedstaaten eingegangen und es werden länderspezifische Steuerbelastungen für die Jahre 2013 bis $2015^{1}$ mit Hilfe des Simulationsprogramms European Tax Analyzer berechnet.

Zunächst werden die wesentlichen steuerlichen Entwicklungen in Deutschland erörtert (Kapitel 2). Anschließend erfolgen eine qualitative Analyse der effektiven Steuerbelastungen bei der Besteuerung von Kapitalgesellschaften und ihren Anteilseignern in den EU- Mitgliedstaaten in den Jahren 2013 bis 2015 (Kapitel 3) sowie eine Diskussion hinsichtlich der Veränderung der Steuerposition Deutschlands (Kapitel 4). Die Ergebnisse werden abschließend in Thesen zusammengefasst (Kapitel 5).

\section{Steuerliche Änderungen in Deutschland 2013 - 2015}

\subsection{Ziele des Koalitionsvertrags}

Die steuerpolitischen Aussagen des im November 2013 vorgelegten Koalitionsvertrags ${ }^{2}$ sind im Vergleich zu früheren Koalitionsverträgen ${ }^{3}$ vage. Die Schwerpunkte liegen auf der Vereinfachung der Steuergesetze und insbesondere der Bekämpfung von aggressiver Steuervermeidung und Steuerhinterziehung. ${ }^{4}$

Während sich für die Einkommensteuer keinerlei Aussagen finden, soll bei der Körperschaftsteuer eine Steuerpflicht für Veräußerungsgewinne aus Streubesitzbeteiligungen geprüft werden, die jedoch nicht zu einer Behinderung von innovativen Start-Ups führen soll. Die Gewerbe- wie auch die Erbschaftsteuer sollen in ihrer bisherigen Form fortgeführt werden, während für die Grundsteuer eine Reform in Betracht gezogen wird. Steuererhöhungen werden nicht explizit ausgeschlossen.

\footnotetext{
${ }^{1}$ Für das Jahr 2015 werden alle Änderungen, die bis zum 31. August 2015 in Kraft getreten sind, berücksichtigt.

2 Vgl. CDU/CSU und SPD, Koalitionsvertrag, 2013, S. 63-66.

${ }^{3}$ Vgl. CDU/CSU und SPD, Koalitionsvertrag, 2005, S. 10-14; CDU/CSU und FDP, Koalitionsvertrag, 2009, S. 68-72.

${ }^{4}$ Diese Bereiche werden im Rahmen dieses Beitrags nicht näher betrachtet. Vgl. hierzu z.B. Spengel, C./Nusser, H., Der Konzern 2015, S. 9-15.
} 


\subsection{Entwicklungen einzelner Steuerarten während der aktuellen Legislaturperiode}

\subsubsection{Erbschaftsteuer}

Noch vor der Bundestagswahl 2013 hatte der BFH Zweifel an der Verfassungsmäßigkeit einzelner Bestimmungen des ErbStG geäußert. ${ }^{5}$ Ende 2014 hat das BVerfG das ErbStG in Teilen für verfassungswidrig erklärt und den Gesetzgeber zu einer Reform der Begünstigungen für Betriebsvermögen ( $§ 13 a, \S 13 b$ ErbStG) bis zum 30. Juni 2016 aufgefordert. ${ }^{6}$ Im Einzelnen kritisierte das BVerfG, dass die Kontrolle der Lohnsumme erst für Betriebe mit 20 und mehr Beschäftigten gilt und die bisherige zulässige Grenze des Verwaltungsvermögens mit 50\% des gesamten Betriebsvermögens insgesamt zu großzügig definiert ist. Zudem sieht das Gericht einen erhöhten Rechtfertigungsbedarf bei der Vererbung großer Betriebsvermögen, die im derzeitigen Gesetz nicht berücksichtigt sind.

Unmittelbar nach dem Urteil wurde bereits verkündet, dass dessen Umsetzung „minimalinvasiv"7 erfolgen würde. Eine grundlegende Reform der Erbschaftsteuer wird somit ausgeschlossen, obwohl entsprechende Reformmodelle bereits seit vielen Jahren diskutiert werden. So würde bei der Einführung eines Flat-Tax Modells die Bemessungsgrundlage verbreitert (z. B. Aufgabe der Vergünstigungen für Betriebsvermögen) und das derzeitige progressive Tarifschema zugunsten eines niedrigen, einheitlichen Steuersatzes aufgegeben. ${ }^{8}$

Das Ziel, möglichst geringe Änderungen an der derzeitigen Konzeption des ErbStG vorzunehmen, wurde bisher weiter verfolgt. Der aktuelle Gesetzentwurf der Bundesregierung ${ }^{9}$ sieht nun neben der Herabsenkung der Kontrollschwelle für die Lohnsummenpflicht auf alle Betriebe mit mehr als drei Beschäftigten ${ }^{10}$ eine Neudefinition des Betriebsvermögensbegriffs zugunsten einer Einzelbetrachtung jedes Vermögensgegenstands vor. Am umstrittensten war und ist immer noch die Ausgestaltung der Regelungen für die Vererbung großer Betriebsvermögen. ${ }^{11}$ Hier sieht der Gesetzentwurf für Betriebsvermögen über 26 Mio. € ein Abschmelzmodell mit einem fallenden, prozentualen Verschonungsabschlag vor oder alternativ eine individuelle Bedürfnisprüfung unter der teilweisen Einbeziehung des sonstigen Vermögens (50\%) des Erben. Bei Erfüllung gewisser qualitativer Merkmale, die bestehende Familienunternehmen schützen sollen,

\footnotetext{
5 Vgl. BFH, Beschluss vom 27.09.2012, BStBl. 2012 II, S. 899.

${ }^{6}$ Vgl. BVerfG, Urteil vom 17.12.2014, BStBl. 2015 II, S. 50.

${ }^{7}$ So der Abteilungsleiter Steuern im BMF Michael Sell bei einer Tagung der Stiftung Familienunternehmen am 9. Januar 2015 und BMF, Neuregelung - Eckwerte, 2015.

${ }^{8}$ Vgl. Wissenschaftlicher Beirat beim BMF, Erbschaftsteuer, 2012; Pahlke, A., ZEV 2015, S. 377-382; Seer, R., GmbHR 2015, S. 118-120; DWS (Hrsg.), Zukunft Erbschaftsteuer, 2015, S. 80, 89 sowie für eine quantitative Analyse einer solchen Reform Houben, H./Maiterth, R., Perspektiven der Wirtschaftspolitik 2010, S. 204-222.

${ }^{9}$ Vgl. Bundesregierung, Gesetzentwurf, 2015.

${ }^{10}$ Für Betriebe, die mehr als 3 aber höchstens 15 Beschäftigte haben, sieht der Gesetzentwurf Erleichterungen bei der zu erreichenden Lohnsumme vor.

${ }^{11}$ Vgl. Krumm, M., FR 2015, S. 481-496.
} 
greift das Abschmelzmodell bzw. die Bedürftigkeitsprüfung erst ab einer Grenze von 52 Mio. €.

Erste Untersuchungen zeigen, dass die Steuerlast für große Unternehmen in allen bisher diskutierten Szenarien ansteigen würde. ${ }^{12}$ Neben den quantitativen Auswirkungen dürfte jedoch auch die Komplexität der bisher schon schwer verständlichen $\S 13 \mathrm{a}$ und $\S 13 \mathrm{~b}$ ErbStG nochmals deutlich zunehmen. ${ }^{13}$ Die verlangte Einzelbetrachtung im Rahmen der Bestimmung des Betriebsvermögens dürfte regelmäßig sehr zeitaufwändig und in Zweifelsfällen schwierig vorzunehmen sein. ${ }^{14}$ Auch die Aufnahme qualitativer Kriterien für die Beurteilung von Gesellschafterverträgen sowie die Bedürftigkeitsprüfung unter Einbeziehung des Privatvermögens des Erben wird für zahlreiche Komplikationen sorgen. ${ }^{15}$ Insgesamt ist es zudem ungewiss, ob die nun beschlossenen Vereinbarungen einer erneuten Prüfung durch das BVerfG standhalten würden. ${ }^{16}$ Die Bundesregierung wird deswegen Schwierigkeiten haben, eine minimalinvasive Umsetzung zu realisieren.

\subsubsection{Grundsteuer/Grunderwerbsteuer}

Bereits seit vielen Jahren gibt es Diskussionen um eine Reform der Grundsteuer. Hauptstreitpunkt ist die Ausgestaltung der Bemessungsgrundlage, die auf veralteten Einheitswerten aus den Jahren 1964 ( $§ 19$ Abs. 1 BewG) bzw. 1935 für die neuen Bundesländer ( $§ 129$ BewG) basiert. ${ }^{17}$ Bereits 2010 hat der BFH ernste Zweifel an der Verfassungsmäßigkeit dieser Einheitswerte für die Zeit nach dem 1.1.2007 geäußert, diese jedoch nicht dem BVerfG zur Entscheidung vorgelegt. ${ }^{18}$ Verschiedenen Ländergruppen haben bereits unterschiedliche Reformmodelle vorgestellt, ${ }^{19}$ deren mögliche fiskalische Auswirkungen kürzlich auch quantitativ für die Stadt Fürth vergleichend untersucht wurden. ${ }^{20}$ Trotz dieser Vorarbeiten und des Appells im Koalitionsvertrag konnten sich die Bundesländer bisher nicht auf eine Reform einigen. Neueste Berichte sprechen zwar von einer möglichen zeitnahen Einigung, jedoch soll die Reform nicht vor 2020 in Kraft treten. ${ }^{21}$ Die jüngste Dynamik dürfte maßgeblich durch eine Vorlage des BFH an das BVerfG am 22.10.2014 beeinflusst worden sein. ${ }^{22}$ Darin stellt der BFH explizit fest, dass die Einheitsbewertung spätestens zum 1.1.2009 als verfassungswidrig anzusehen ist. Es

\footnotetext{
12 Vgl. Heinemann, F. et al., Quantitative Auswirkungen Erbschaftsteuer, 2015.

13 Vgl. Erkis, G., DStR 2015, S. 1415.

14 Vgl. Hannes, F., ZEV 2015, S. 372; Korezkij, L., DStR 2015, S. 1650.

15 Vgl. Erkis, G., DStR 2015, S. 1413; Korezkij, L., DStR 2015, S. 1652.

${ }^{16}$ So auch Hey, J., DB 2015, Heft 16, S. M5; Kirchhof, G., DStR 2015, S. 1473-1481; Roth, H.-P., SteuK 2015 , S. 292.

17 Vgl. dazu ausführlich Halaczinsky, R., DStR-Beihefter 2014, S. 139-148.

18 Vgl. BFH, Urteil vom 30.06.2010, BStBl. 2010 II, S. 897.

${ }^{19}$ Vgl. für einen Überblick über die Reformmodelle Wissenschaftlicher Beirat beim BMF, Grundsteuerreform, 2010; Becker, J., BB 2011, S. 535-539; Spengel, C. et al., DB 2011, S. 10-14; Spengel, C., StBW 2012, S. 31-36.

${ }^{20}$ Vgl. Nehls, D./Scheffler, W., ifst-Schrift 503, 2015.

21 Vgl. Keller, D., Südwest Presse 06.08.2015.

22 Vgl. BFH, Beschluss vom 22.10.2014, BStBl. 2014 II, S. 957.
} 
bleibt abzuwarten, ob sich der Gesetzgeber bereits vor einem eventuellen Urteil des BVerfG zu einer Reform entschließen kann. ${ }^{23}$

Den Schritt der verfassungsrechtlichen Prüfung hat die Grunderwerbsteuer bereits hinter sich. In einem kürzlich ergangenen Urteil wurde die Verwendung der Ersatzbemessungsgrundlage nach $\S 8$ Abs. 2 GrEStG für nicht verfassungsgemäß erklärt. ${ }^{24}$ Diese Ersatzbemessungsgrundlage kommt regelmäßig bei Übertragungen von Grundbesitz im Rahmen von Unternehmensübernahmen oder -umstrukturierungen zur Anwendung. Die für die Bewertung anzuwendenden $§ \S 138 f f$. BewG hatte das BVerfG bereits 2006 für die Erbschaftsteuer als unsachgemäß kritisiert. ${ }^{25} \mathrm{Im}$ jetzigen Urteil kritisierte das BVerfG die sehr ungleichen Ergebnisse der Bewertung nach der Ersatzbemessungsgrundlage im Vergleich zum Regelfall (Gegenleistung) nach § 8 Abs. 1 GrEStG. Nach Aussagen des BMF betrifft die Ersatzbemessungsgrundlage nur einen sehr kleinen Teil aller Fälle der GrESt (0,8\%). Das Aufkommen dieser Fälle summiert sich jedoch auf einen jährlichen dreistelligen Millionenbetrag. ${ }^{26}$ Das BVerfG hat dem Gesetzgeber wie bei der Erbschaftsteuer eine Frist bis zum 30. Juni 2016 für eine Neuregelung eingeräumt und eine rückwirkende Anwendung ab dem 1. Januar 2009 gefordert. ${ }^{27}$ Mit Verweis auf verfahrensrechtliche Hindernisse ( $§ 176 \mathrm{AO}$ ) sieht der derzeitige Gesetzentwurf eine rückwirkende Anwendung lediglich für bisher noch nicht veranlagte Fälle vor. ${ }^{28}$ Die Bewertung soll zukünftig wie im Rahmen der Erbschaftsteuer nach $§ \S 157 f f$. BewG erfolgen.

Neben diesen konzeptionellen Problemen wird auch die wirtschaftliche Belastung von Grundsteuer und Grunderwerbsteuer durch die stetig steigenden Hebesätze bzw. Steuersätze größer. So stieg der durchschnittliche Hebesatz der Grundsteuer (Grundsteuer B) aller Gemeinden mit mehr als 50.000 Einwohnern in Deutschland von 2008 bis 2015 von $494 \%$ auf 563\% an. ${ }^{29}$ Bei der Grunderwerbsteuer, deren Steuersatz seit einigen Jahren eigenständig von den Bundesländern festgelegt wird, ist gerade in den beiden letzten Jahren eine spürbare Erhöhung zu verzeichnen. ${ }^{30}$ Der noch bis 2006 bundeseinheitlich geltende Satz von 3,5\% ist heute nur noch in Bayern und Sachsen anwendbar. In vier Bundesländern (Brandenburg, Nordrhein-Westfalen, Saarland, Schleswig-Holstein) beträgt der Steuersatz mittlerweile 6,5\%, was im Vergleich zum Jahr 2006 beinahe einer Verdoppelung der Steuerbelastung entspricht.

\footnotetext{
23 So auch Scheffler, W., DB 2015, Heft 8, S. M5.

24 Vgl. BVerfG, Beschluss vom 23.06.2015, BGBl. 2015 I, S. 1423.

25 Vgl. BVerfG, Beschluss vom 07.11.2006, BGBl. 2007 I, S. 194.

26 Vgl. BVerfG, Beschluss vom 23.06.2015, BGBl. 2015 I, S. 1423, Rn. 34.

27 Vgl. BVerfG, Beschluss vom 23.06.2015, BGBl. 2015 I, S. 1423, Rn. 91-92.

28 Vgl. BT-Drucksache 18/6094, S. 90-91.

${ }^{29}$ Vgl. Andrae, K., ifst-Schrift 504, 2015, S. 37 sowie für das Jahr 2015 eine Sonderauswertung des DIHKRealsteuervergleichs. Vgl. DIHK, Realsteuervergleich, 2015.

${ }^{30}$ Vgl. Ministerium der Finanzen Brandenburg, Übersicht GrESt-Sätze, 2015.
} 


\subsubsection{Gewerbesteuer}

Nach dem Scheitern einer umfassenden Reform der Gewerbesteuer im Rahmen der Gemeindefinanzkommission $2011^{31}$ hat man sich für die aktuelle Legislaturperiode nicht erneut das Ziel einer Systemumstellung gesetzt. Einzelne Änderungen am bestehenden GewSt-Gesetz könnten jedoch von der Rechtsprechung gefordert werden. So will das BVerfG noch in diesem Jahr über die Hinzurechnung von pauschalen Finanzierungsanteilen nach $\S 8$ Nr. 1 a), d) und e) GewStG entscheiden. ${ }^{32}$ Der BFH hat in dieser Frage die Verfassungsmäßigkeit der Vorschriften trotz Bedenken in der Literatur ${ }^{33}$ bisher bejaht. $^{34}$

Viel bedeutender als die auch hier bestehenden konzeptionellen Mängel ist jedoch die langsam, aber stetig steigende wirtschaftliche Belastung der Unternehmen durch die Gewerbesteuer. Seit der Versagung des Betriebsausgabenabzugs im Rahmen der Unternehmenssteuerreform 2008 ist die Gewerbesteuer für viele Kapitalgesellschaften von höherer Bedeutung als die Körperschaftsteuer. So sind die durchschnittlichen GewStHebesätze in Gemeinden mit mehr als 50.000 Einwohnern zwischen 2008 und 2015 von $432 \%$ auf $447 \%$ gestiegen. ${ }^{35}$ Die Tarifbelastung der Gewinne von Kapitalgesellschaften (KSt, GewSt, SolZ) hat sich somit von 30,95\% auf 31,47\% erhöht.

\subsubsection{Körperschaftsteuer}

Nach Einführung einer Steuerpflicht auf Streubesitzdividenden im Jahr 2013 aus europarechtlichen Gründen, ${ }^{36}$ hatte der Bundesrat schon 2014 die Einführung einer analogen Regelung für Veräußerungsgewinne gefordert, deren Prüfung auch von der Bundesregierung zugesagt wurde. ${ }^{37}$ Die Einführung soll laut Koalitionsvertrag jedoch zu keinen Behinderungen von Investitionen in Start-Up Unternehmen führen. ${ }^{38}$ Ein kürzlich veröffentlichter Diskussionsentwurf des BMF bleibt hinter diesen Erwartungen zurück. ${ }^{39}$ Dieser sieht zunächst eine generelle Steuerpflicht für Veräußerungsgewinne vor, sofern der Anteil weniger als $10 \%$ beträgt. Für Investitionen in Start-Ups soll eine Ermäßigung der auf den Veräußerungsgewinn entrichteten Körperschaftsteuer eingeführt werden, die auf höchstens 30\% der Anschaffungskosten des Anteils beschränkt ist. Diese Grenze könnte insbesondere bei erfolgreichen Start-Ups mit einer sehr hohen Wertsteigerung

\footnotetext{
31 Vgl. Gemeindefinanzkommission - AG "Kommunalsteuern", Abschlussbericht, 2011.

32 Vgl. BVerfG, Jahresvorschau, 2015, 1 BvL 8/12 aufgrund einer Vorlage des FG Hamburg. Vgl. FG Hamburg, Beschluss vom 29.2.2012, DStRE 2012, S. 478.

33 Vgl. Grünwald, K./Friz, F., DStR 2012, S. 2106-2111; Ritzer, C., DStR 2013, S. 558-565.

34 Vgl. BFH, Beschluss vom 16.10.2012, BStBl. 2013 II, S. 30; BFH, Urteil vom 04.06.2014, BStBl. 2015 II, S. 289. Kritisch dazu Keß, T., FR 2013, S. 188-189.

35 Vgl. Andrae, K., ifst-Schrift 504, 2015, S. 29 sowie für das Jahr 2015 eine Sonderauswertung des DIHKRealsteuervergleichs. Vgl. DIHK, Realsteuervergleich, 2015.

36 Vgl. dazu Benz, S./Jetter, J., DStR 2013, S. 489-496; Watrin, C./Eberhardt, D., IStR 2013, S. 814-820.

${ }^{37}$ Vgl. BR-Drucksache 291/14 und Bundesregierung, Protokollerklärung, 2014.

${ }^{38}$ Vgl. CDU/CSU und SPD, Koalitionsvertrag, 2013, S. 64.

${ }^{39}$ Vgl. BMF, Investmentbesteuerung, 2015, S. 31-33.
} 
schnell erreicht sein. Nach dem derzeitigen Vorschlag müssten für die Ermäßigung zusätzlich sechs qualitative Bedingungen kumulativ erfüllt werden. ${ }^{40}$

Auch die Zinsschranke nach $\S 4 \mathrm{~h}$ EStG, die insbesondere für multinationale Konzerne aufgrund der verschärften Bedingungen des $\S 8$ a KStG bedeutsam ist, könnte dem Gesetzgeber noch in dieser Legislaturperiode Probleme bereiten. ${ }^{41}$ Der BFH hat bereits Ende 2013 ernste Zweifel an der Verfassungsmäßigkeit der Zinsschranke geäußert. ${ }^{42}$ Mittlerweile sind zwei weitere Verfahren zu dieser Frage beim BFH anhängig und es ist noch im Jahr 2015 mit einer Entscheidung zu rechnen. ${ }^{43}$

\subsubsection{Einkommensteuer}

Im Bereich der Einkommensteuer wird in erster Linie über eine Milderung der kalten Progression intensiv diskutiert. ${ }^{44}$ Nachdem dieses Vorhaben noch in der vorherigen Legislaturperiode am Widerstand des Bundesrates gescheitert war, ${ }^{45}$ hat man sich kürzlich zumindest auf eine Rechtsverschiebung der ESt-Tarife um 1,48\% ab 2016 verständigt. ${ }^{46}$ Diese Tarifverschiebung soll die Inflationswirkung der Jahre 2014 und 2015 ausgleichen. ${ }^{47}$ Es ist nicht zu erwarten, dass man sich in der verbleibenden Legislaturperiode auf eine langfristige Lösung verständigt, die z. B. einen automatischen jährlichen Inflationsausgleich ermöglicht, wie er in vielen anderen Ländern vorzufinden ist. Die bisherige Fixierung der politischen Diskussion auf die Änderung des Tarifverlaufs erfasst das Problem der kalten Progression nur unvollständig. ${ }^{48}$

Ein weiteres Problem stellt die Benachteiligung der Besteuerung gewerblicher Einkünfte im Vergleich zu anderen Einkunftsarten dar, die unmittelbar aus den steigenden GewStHebesätzen folgt. Der Anrechnungsmechanismus des § 35 EStG beschränkt den Anrechnungshöchstbetrag bei 380\% des Gewerbesteuermessbetrags. Eine Auswertung der vorläufigen GewSt-Hebesätze für das Jahr 2015 zeigt, dass mittlerweile 162 von insgesamt 182 Kommunen mit mehr als 50.000 Einwohnern einen Hebesatz von über $380 \%$ haben. ${ }^{49}$ Bei Kommunen mit mehr als 20.000 Einwohnern liegt dieser Anteil bei $63 \% .{ }^{50}$ In immer mehr Kommunen kommt es somit zu einer Definitivbelastung der gewerblichen Einkünfte mit Gewerbesteuer. Es könnte daher überlegt werden, den seit 2008 unverändert geltenden Anrechnungshöchstbetrag von 380\% zu erhöhen, um das ursprüngli-

\footnotetext{
${ }^{40}$ Vgl. BMF, Investmentbesteuerung, 2015, S. 31.

41 Vgl. zur Kritik in der Literatur Heuermann, B., DStR 2013, S. 1-5; Prinz, U., DB 2014, S. 1102-1103.

42 Vgl. BFH, Beschluss vom 18.12.2013, BStBl. 2014 II, S. 947.

${ }^{43}$ Vgl. BFH, Entscheidungsvorschau, 2015. Die anhängigen Verfahren werden unter den Aktenzeichen I R 2/13 und I R 57/13 geführt. Am 18. August fand im Verfahren I R 2/13 bereits eine mündliche Anhörung statt.

44 Vgl. z. B. SPD, Kalte Progression, 2014; CDU, Kalte Progression, 2014.

45 Vgl. BR-Drucksache 201/12.

46 Vgl. BT-Drucksache 18/5244 sowie Zustimmung des Bundesrats BR-Drucksache 281/15.

47 Vgl. BT-Drucksache 18/5244, S. 31.

48 Vgl. z. B. Eichfelder, S./Hechtner, F., DStZ 2013, S. 227-242; Hechtner, F., StuW 2014, S. 132-144;

Djanani, C./Grossmann, T., StuW 2015, S. 33-51.

${ }^{49}$ Eigene Auswertung des DIHK-Realsteuervergleichs. Vgl. DIHK, Realsteuervergleich, 2015.

${ }^{50}$ Eigene Auswertung des DIHK-Realsteuervergleichs. Vgl. DIHK, Realsteuervergleich, 2015.
} 
che Ziel einer gleichmäßigen Belastung von gewerblichen Einkünften und anderen Einkunftsarten zu erreichen. ${ }^{51}$

\subsection{Zwischenfazit}

Die Gesamtbetrachtung der Entwicklung des deutschen Steuersystems zur Halbzeit der aktuellen Legislaturperiode fällt eher ernüchternd aus. In beinahe jeder Steuerart gibt es gravierende konzeptionelle Mängel, die von der aktuellen Bundesregierung nicht proaktiv angegangen werden. Auf das originäre Gestaltungsrecht der Politik wird zunehmend verzichtet, da die meisten Reformimpulse mittlerweile von der Rechtsprechung des BFH und BverfG ausgehen.

Insgesamt wurden die wenig ambitionierten Ziele des Koalitionsvertrags bisher nicht umgesetzt. Aktuelle Reformvorhaben (Erbschaftsteuer, Steuerpflicht für Veräußerungsgewinne aus Streubesitzbeteiligungen) lassen eher eine Verkomplizierung des Steuerrechts erwarten und widersprechen damit dem Zielen des Koalitionsvertrags. Insbesondere werden verschärfende Regelungen wie die Steuerpflicht für Veräußerungsgewinne die Rahmenbedingungen für Investitionen in innovationsfreudige Start-Ups eher verschlechtern. Hinzu kommt die steigende tarifliche Steuerbelastung in vielen Steuerarten (Grundsteuer, Grunderwerbsteuer, Gewerbesteuer). Auch die seit langem bekannte Problematik der kalten Progression wurde nur kurzfristig gelöst und aufgrund der steigenden GewSt-Hebesätze ergibt sich eine zunehmend ungleiche Besteuerung von gewerblichen und nicht-gewerblichen Einkünften.

\section{Qualitative Analyse der Steueränderungen in der Europäischen Union}

\section{3-2015}

Nach dem Überblick über die Entwicklungen des deutschen Steuersystems werden in einem nächsten Schritt die steuerlichen Änderungen in den anderen EU-Mitgliedstaaten im Zeitraum 2013 bis 2015 dargestellt und analysiert. ${ }^{52}$ Die Analyse beschränkt sich auf die laufende Besteuerung von Kapitalgesellschaften und ihrer Anteilseigner.

\subsection{Besteuerung von Kapitalgesellschaften (Unternehmensebene)}

\subsubsection{Entwicklung der effektiven Tarifbelastung (KSt-Sätze zuzüglich Zuschlagsteuern und} lokalen Gewinnsteuern)

Seit vielen Jahren sinken die Körperschaftsteuersätze und somit auch die Taribelastungen der Unternehmensgewinne in den EU-Mitgliedstaaten. Diese Entwicklung lässt sich mit einem zunehmend intensiveren Steuerwettbewerb um Investitionen von Unternehmen begründen und ist empirisch gut belegt. ${ }^{53}$ Für den Beginn des Unter-

\footnotetext{
51 Siehe dazu BT-Drucksache 16/4841, S. 63; Jacobs, O. H. et al., Unternehmensbesteuerung, 2015, S. 153.

52 Die präsentierten Ergebnisse beruhen auf einer umfassenden Auswertung der Datenbank des International Bureau for Fiscal Documentation (IBFD).

53 Vgl. z. B. Devereux, M. P./Loretz, S., National Tax Journal 2013, S. 745-773; Redoano, M., European Journal of Political Economy 2014, S. 351-371.
} 
suchungszeitraums (2013) ließ sich jedoch kurzzeitig eine gegenläufige Bewegung erkennen, nachdem im Zuge der Finanz- und Wirtschaftskrise mehrere Mitgliedstaaten ihre Körperschaftsteuersätze (Zypern, Griechenland, Slowakische Republik) oder Zuschlagsteuern (Luxemburg, Portugal) zum 1. Januar 2013 erhöht hatten. ${ }^{54}$ Diese Entwicklung hat sich für die Jahre 2014 und 2015 jedoch nicht fortgesetzt, wie Tabelle $1 \mathrm{zu}$ entnehmen ist. Vielmehr ist eine Rückkehr zum vormaligen Trend zu erkennen, da in insgesamt sieben Mitgliedstaaten der KSt-Satz gesenkt wurde. Nur in Griechenland wurde der KSt-Satz auf Druck der internationalen Geldgeber im Jahr 2015 von 26\% auf 29\% erhöht. 55

\section{Tabelle 1: Tarifbelastungen von Unternehmensgewinnen (KSt-Sätze zuzüglich Zuschlagsteuern und lokalen Gewinnsteuern in \%) 2013-201556}

\begin{tabular}{cccc} 
EU-Mitgliedstaat & $\mathbf{2 0 1 3}$ & $\mathbf{2 0 1 4}$ & $\mathbf{2 0 1 5}$ \\
\hline Dänemark & 25,0 & $\mathbf{2 4 , 5}$ & $\mathbf{2 3 , 0}$ \\
Deutschland & $\mathbf{3 1 , 4}$ & $\mathbf{3 1 , 4}$ & $\mathbf{3 1 , 5}$ \\
Estland & 21,0 & 21,0 & $\mathbf{2 0 , 0}$ \\
Finnland & 24,5 & $\mathbf{2 0 , 0}$ & 20,0 \\
Griechenland & 26,0 & 26,0 & $\mathbf{2 9 , 0}$ \\
Großbritannien & 23,0 & $\mathbf{2 1 , 0}$ & $\mathbf{2 0 , 0}$ \\
Portugal & 30,0 & $\mathbf{2 8 , 0}$ & $\mathbf{2 6 , 0}$ \\
Slowakische Republik & 23,0 & $\mathbf{2 2 , 0}$ & 22,0 \\
Spanien & 35,3 & 35,3 & $\mathbf{3 3 , 4}$ \\
EU-28 Durchschnitt & $\mathbf{2 3 , 4}$ & $\mathbf{2 3 , 1}$ & $\mathbf{2 2 , 9}$
\end{tabular}

Finnland hat den KSt-Satz in 2014 von 24,5\% auf 20\% gesenkt und damit die größte Reduzierung aller EU-Mitgliedstaaten vollzogen. Weitere Länder mit erheblichen Reduzierungen des KSt-Satzes sind Portugal und Großbritannien, die beide schrittweise den KSt-Satz von 25\% bzw. 23\% (2013) auf 21\% bzw. 20\% (2015) gesenkt haben. Mit dem neuen KSt-Satz von 20\% in Großbritannien, der seit 1. April 2015 zur Anwendung kommt, ist nun das vorläufige Ziel einer langjährigen schrittweisen Reduzierung des

\footnotetext{
54 Vgl. ausführlich Spengel, C. et al., DB 2014, S. 1097.

55 Vgl. Europäische Kommission, List of Prior Actions, 2015.

56 Zuschlagsteuern sind in Deuschland, Portugal und Spanien zu beachten. In Deutschland wird neben dem SolZ von 5,5\% der Durchschnitt der GewSt-Hebesätze für Gemeinden über 50.000 Einwohner einbezogen, siehe Andrae, K., ifst-Schrift 504, 2015 sowie DIHK, Realsteuervergleich, 2015. Eine lokale Gewinnsteuer in Portugal (derrama estadual) ist progressiv ausgestaltet. Sie wurde 2014 um eine dritte Progressionsstufe (7\%) erhöht, die jedoch erst für Einkommen über 35 Mio. Euro zur Anwendung kommt. In der Tabelle wird weiterhin der Steuersatz der zweiten Progressionsstufe (5\%) verwendet. Der lokale Gewinnsteuersatz in Spanien beträgt im Durchschnitt 7,5\%. Die Steuer (imposte sobre actividades) ist von der Bemessungsgrundlage der Körperschaftsteuer abzugsfähig, weshalb sich eine geringere effektive Tarifbelastung ergibt. Der EU-28 Durchschnitt ergibt sich aus Spengel, C. et al., Effective Tax Levels Using the Devereux/Griffith Methodology, 2014, S. B-1 und zusätzlichen eigenen Berechnungen.
} 
KSt-Satzes von 28\% im Jahr 2010 erreicht worden. ${ }^{57}$ Eine weitere schrittweise Reduzierung gibt es in Dänemark von 25\% (2013) auf 23\% (2015). Interessant ist die Entwicklung in der Slowakischen Republik, die mit der Reduzierung des KSt-Satzes auf 22\% die erst in 2013 erfolgte Erhöhung von 19\% auf 23\% teilweise zurückgenommen hat. Die in Estland vorgenommene Steuersatzsenkung von $21 \%$ auf $20 \%$ betrifft ausschließlich ausgeschüttete Gewinne, da thesaurierte Gewinne weiterhin steuerbefreit sind. Spanien hat nach der Reduktion des KSt-Satzes von 30\% auf 28\% in 2015 eine weitere Reduzierung ab 2016 auf 25\% angekündigt.

Die leicht ansteigende Tarifbelastung in Deutschland, die durch die steigenden Hebesätze der Gewerbesteuer ausgelöst wird, widerspricht dem EU-weiten Trend. Dies lässt sich neben den Steuersatzsenkungen in einzelnen Ländern auch an dem sinkenden Durchschnitt der Tarifbelastung aller 28 EU-Mitgliedstaaten erkennen, der von 23,4\% auf 22,9\% für das Jahr 2015 sinkt.

\subsection{2 Änderungen bei der Bemessungsgrundlage der Körperschaftsteuer}

Neben der Reduzierung der Steuersätze hat es bisher eine klare Tendenz zu einer Verbreiterung der Bemessungsgrundlage gegeben, die sich auch im Zeitraum 2013-2015 fortsetzt. Eine Übersicht über die einzelnen Maßnahmen sowie vergleichbare Regelungen in Deutschland gibt Tabelle 2.

Tabelle 2: Veränderungen der KSt-Bemessungsgrundlage 2013-2015 in der EU und vergleichbare Regelungen in Deutschland

\begin{tabular}{|c|c|c|c|c|c|}
\hline EU-Mitgliedstaat & Jahr & & & & \\
\hline \multicolumn{2}{|c|}{ Beschränkung des Zinsabzugs } & $\begin{array}{l}\text { Berücksichtigtes } \\
\text { Fremdkapital }\end{array}$ & $\begin{array}{l}\text { Freigrenze } \\
(\text { in Mio. } €)\end{array}$ & $\begin{array}{l}\text { EBITDA- } \\
\text { Grenze }\end{array}$ & $\begin{array}{l}\text { Vortrag } \\
\text { möglich? }\end{array}$ \\
\hline Deutschland & & Gesamt & 3,0 & $30 \%$ & ja \\
\hline Frankreich & 2013 & Gesamt & 3,0 & $\begin{array}{c}85 \% \\
75 \%(2014)\end{array}$ & nein \\
\hline Finnland & 2014 & $\begin{array}{l}\text { verbundene An- } \\
\text { teilseigner }\end{array}$ & $\begin{array}{l}0,5 \text { (Freibe- } \\
\text { trag) }\end{array}$ & $25 \%$ & ja \\
\hline Griechenland & 2014 & Gesamt & 5,0 & $\begin{array}{c}60 \% \\
50 \%(2015)\end{array}$ & ja \\
\hline Polen & 2015 & \multicolumn{4}{|c|}{$\begin{array}{l}\text { Zinsabzug nur, sofern das Eigenkapital eines Anteilseigners } \\
\text { höher ist als das gewährte Fremdkapital }\end{array}$} \\
\hline Portugal & 2013 & Gesamt & 3,0 & $\begin{array}{c}70 \% \\
60 \%(2014) \\
50 \%(2015)\end{array}$ & ja \\
\hline Slowakische Republik & 2014 & $\begin{array}{l}\text { verbundene An- } \\
\text { teilseigner }\end{array}$ & - & $25 \%$ & nein \\
\hline
\end{tabular}

\footnotetext{
${ }^{57}$ Großbritannien hat bereits weitere Reduzierungen angekündigt. Nach derzeitigen Planungen soll der KSt-Satz in 2017 auf 19\% sinken und ab 2020 18\% betragen.
} 


$\begin{array}{cccc}\text { Beschränkung von Verlustvorträgen } & \begin{array}{c}\text { Max. nutzbarer } \\ \text { Vortrag } \\ \text { 1 Mio. } €+60 \%\end{array} & \begin{array}{c}\text { Max. zeitliche } \\ \text { Nutzung in } \\ \text { Jahren }\end{array} \\ \text { Deutschland } & 2013 & 1 \text { Mio. } €+50 \% & - \\ \text { Frankreich } & 2015 & - & - \\ \text { Ungarn } & 2014 & 70 \% & - \\ \text { Litauen } & 2015 & 70 \% & 12 \\ \text { Portugal } & 2014 & - & 4 \\ \text { Slowakische Republik } & 2013 & 50 \% & - \\ \text { Slowenien } & & \end{array}$

\section{Abschreibungsregelungen}

Deutschland

lineare Abschreibung seit 2011 verpflichtend

$\begin{array}{cc}\text { Griechenland } & 2013 \\ & 2015 \\ \text { Spanien } & 2015\end{array}$

Abschaffung der degressiven Abschreibungsmöglichkeiten

Abschaffung der degressiven Abschreibung für Gebäude

verpflichtende lineare Abschreibung von immateriellen Wirtschaftsgütern mit $5 \%$ p.a.

\section{Fiktiver Zinsabzug für Eigenkapital (Allowance for Corporate Equity, ACE)}

$\begin{array}{cc}\text { Belgien } & 2014 \\ \text { Zypern } & 2015 \\ \text { Litauen } & 2014 \\ \text { Italien } & 2013\end{array}$
Abschaffung der Vortragsmöglichkeit; Begrenzung des Zinssat- zes und Einführung einer Mindeststeuer

ACE für ab 2015 zugeführtes Eigenkapital; Zinssatz orientiert sich an langfristigen Staatsanleihen $+3 \%$

ACE ersatzlos abgeschafft

Erhöhung des maßgeblichen Zinses von 3\% (2013), auf 4\%

(2014) und 4,5\% (2015)

Viele neue Regelungen zur Beschränkung des Zinsabzugs orientieren sich in ihrer Ausgestaltung an der deutschen Zinsschranke nach § 4h EStG. Die zulässigen prozentualen EBITDA-Grenzen und Freigrenzen sind in den meisten Fällen (z. B. Frankreich, Griechenland) derzeit höher als in Deutschland. Portugal und Griechenland planen jedoch eine schrittweise Absenkung der EBITDA-Grenze auf 30\% bis 2017 und ab 2016 beträgt die Freigrenze in Griechenland wie in Deutschland 3 Mio. €. In der Gesamtbetrachtung aus derzeitiger EBITDA-Grenze und zulässigem Freibetrag ist die deutsche Regelung im Vergleich mit Frankreich, Griechenland und Portugal eher als restriktiv anzusehen. Ein solcher Vergleich ist mit den neueingeführten Regelungen in Finnland, Polen und der Slowakischen Republik nicht möglich, da sie nur das Kapital von verbundenen Anteilseignern einbeziehen. Mit der Slowakischen Republik und Frankreich haben zwei Länder diese Beschränkungen ohne die Gewährung einer Vortragsmöglichkeit für nicht abzugsfähige Zinsen eingeführt. In dieser Hinsicht sind die eingeführten Regelungen restriktiver als in Deutschland. Insgesamt haben im Jahr 2015 nun bereits 20 EU-Mitgliedstaaten Zinsabzugsbeschränkungen in ihren Steuergesetzen implementiert. 
Zudem wird die Nutzung von Verlustvorträgen zunehmend zeitlich oder betragsmäßig begrenzt. Neue zeitliche Beschränkungen sind in der Slowakischen Republik (vier Jahre statt sieben Jahre) und in Ungarn zu finden (fünf Jahre statt unbegrenzt). Bereits 2013 hat Frankreich die Grenze für die Nutzung von Verlustvorträgen von über 1 Mio. € von 60\% auf 50\% gesenkt. Slowenien und Litauen haben im Jahr 2013 bzw. 2014 eine betragsmäßige Begrenzung des Verlustvortrags von 50\% bzw. 70\% eingeführt. In Portugal gab es eine leichte Reduktion dieser Grenze von 75\% auf 70\% und der Vortragszeitraum wurde von fünf auf zwölf Jahre erhöht. Die derzeitige deutsche Regelung ist im EUVergleich zumeist vorteilhaft, da ein zeitlich unbegrenzter Verlustvortrag möglich ist. Auch die betragsmäßige Begrenzung ist weniger restriktiv als viele der Neuregelungen, da erst für Verluste über 1 Mio. € eine prozentuale Beschränkung greift.

Hinsichtlich der Modifizierung von Abschreibungsmöglichkeiten lassen sich im Untersuchungszeitraum nur geringfügige Änderungen in Spanien und der Slowakischen Republik feststellen. Griechenland hat bereits 2013 sämtliche Möglichkeiten einer degressiven Abschreibung abgeschafft. Damit nähern sich diese Mitgliedstaaten den Regelungen in Deutschland an, die seit 2011 ausschließlich eine lineare Abschreibung vorsehen.

Eine hohe Dynamik gibt es bei Regelungen, die einen fiktiven Zinsabzug auf das Eigenkapital (Allowance for Corporate Equity, ACE) vorsehen. ACE-Regime bezwecken im Grundsatz, die Diskriminierung der Eigen- gegenüber der Fremdkapitalfinanzierung auf Unternehmensebene abzubauen. Bisher waren ACE-Regime in Belgien, Litauen und Italien anzutreffen. Zypern hat 2015 ein solches Regime eingeführt. Während Belgien und Litauen den Zinsabzug auf den gesamten Betrag des Eigenkapitals erlauben, ist dies in Italien und auch in Zypern nur für den Zuwachs des Eigenkapitals seit dem Einführungsjahr 2011 bzw. 2015 erlaubt. Litauen hat in 2014 sein ACE-Regime abgeschafft. In Belgien wurden bereits 2013 erste Restriktionen eingeführt, nach dem der fiktive Zins ${ }^{58}$ nicht mehr als 3\% betragen kann und in einem Jahr nicht mehr als einen Prozentpunkt schwanken darf. 2014 hat Belgien zusätzlich eine Mindestbesteuerung eingeführt, sofern die ausgeschüttete Dividende größer als das steuerpflichtige Einkommen ist. Nur Italien hat mit der Erhöhung des maßgeblichen Zinssatzes von 3\% (2013) auf 4,5\% (2015) die Bedeutung der ACE erhöht. Für Zypern wurde bisher noch kein offizieller Zinssatz veröffentlicht; dieser soll sich an langfristigen Staatsanleihen und einem pauschalen Zuschlag von 3\% orientieren.

Frankreich und Italien haben zudem steuerliche Maßnahmen eingeführt, die direkt die Schaffung oder Erhaltung von Arbeitsplätzen belohnen. In Frankreich wurde 2013 eine Steuergutschrift (crédit d'impôt pour la compétitivité et l'emploi) eingeführt, die sich auf 4\% der Bruttolohnsumme beläuft und 2014 auf 6\% erhöht wurde. Im Rahmen der italienischen Wertschöpfungsteuer IRAP (imposta regionale sulle attività produttive) war bis 2013 kein Abzug von Lohnkosten möglich. 2013 und 2014 wurden Pauschalen von

${ }^{58}$ Grundsätzlich wird dieser abgeleitet aus der Rendite zehnjähriger belgischer Staatsanleihen. 
$4.500 €$ bzw. $7.500 €$ pro festangestelltem Mitarbeiter gewährt. Seit 2015 können Lohnkosten für festangestellte Mitarbeiter unbegrenzt von der Bemessungsgrundlage der IRAP abgezogen werden.

\subsection{Besteuerung auf Ebene der Anteilseigner (natürliche Personen)}

Die Senkung von Körperschaftsteuersätzen kann trotz einer Verbreiterung der Bemessungsgrundlage zu Steuermindereinahmen führen. Als Gegenmaßnahme könnten Mitgliedstaaten die Steuersätze für Dividenden oder Zinseinkünfte auf Anteilseignerebene erhöhen, um die fiskalischen Auswirkungen einer solchen Senkung zu reduzieren. Eine solche Entwicklung lässt sich für den Zeitraum 2013 bis 2015 bei zwei Ländern eindeutig feststellen.

So hat Finnland nach der Senkung des KSt-Satzes von 24,5\% auf 20\% in 2014 die Besteuerung von Kapitaleinkünften in vielerlei Hinsicht verschärft. Zunächst wurde in 2014 bei den Dividendeneinkünften der festgeschriebene Anteil, der als Einkommen den regelmäßig günstigeren, regulären Einkommensteuersätzen unterliegt, von $30 \%$ auf $25 \%$ reduziert. ${ }^{59}$ Die übrigen Dividenden werden als Kapitaleinkünfte qualifiziert und es wird zunächst ein Freibetrag von $150.000 €$ für Dividenden gewährt. Darüber hinaus gehende Dividenden unterliegen zusammen mit allen Zinseinkünften einem zweistufigen progressiven Tarif, bei dem seit 2015 bereits ab $30.000 €$ ein Steuersatz von 33\% zur Anwendung kommt (2013: $50.000 €$ und Steuersatz von 32\%). Eine ähnliche Entwicklung findet sich in Dänemark. Dort wurde der Eingangssteuersatz für Kapitaleinkünfte und sonstige Einkünfte in zwei Schritten von 5,83\% auf 8,08\% in 2015 erhöht.

Weitere Staaten, die die Besteuerung von Kapitaleinkünften im Untersuchungszeitraum erhöht haben, sind Italien, Irland und Luxemburg. Italien hat seit 1. Juli 2014 die finale Quellensteuer auf Dividenden aus Minderheitsbeteiligungen ${ }^{60}$ sowie für Zinseinkünfte aus Bankguthaben von 20\% auf 26\% erhöht. In Luxemburg wurde aus fiskalischen Gründen eine neue Steuer eingeführt, die pauschal 0,5\% des gesamten Einkommens beträgt. Irland hat den Steuersatz für Zinseinkünfte aus Bankguthaben (deposit interest retention tax) 2014 von 33\% auf 41\% erhöht. Zusätzlich wurde der Spitzensteuersatz einer weiteren Steuer auf das Einkommen (universal social charge) leicht von 10\% auf $11 \%$ angehoben.

In Griechenland, den Niederlanden und Litauen wurden sowohl Steuersenkungen wie auch Steuererhöhungen für einzelne Arten von Kapitaleinkünften vollzogen. Griechenland hat 2014 den Steuersatz für Dividenden von 25\% auf 10\% gesenkt. Zugleich wurde in 2015 eine neue Progressionsstufe bei der Solidaritätsabgabe eingeführt, die ab einem Einkommen von $500.000 €$ greift. In Litauen wurde der Steuersatz für Dividenden von $20 \%$ auf 15\% reduziert. Gleichzeitig wurde jedoch 2014 eine Steuer von 15\% auf Zins-

\footnotetext{
${ }^{59}$ Dieser Betrag darf maximal 9\% (2013) bzw. 8\% (seit 2014) des Marktwerts der Beteiligung betragen.

${ }^{60}$ Eine solche ist gegeben, sofern die Anteile an den Stimmrechten weniger als $20 \%$ oder am Gesamtkapital weniger als $25 \%$ betragen.
} 
einkünfte eingeführt, die zuvor von der Besteuerung ausgenommen waren. In den Niederlanden gab es eine einmalige Vergünstigung für Dividenden bis $250.000 € \mathrm{im}$ Jahr 2014. Statt 25\% unterlagen Dividenden unterhalb dieser Grenze nur einem reduzierten Steuersatz von 22\%. Demgegenüber wurden die Steuersätze für Zinsen aus Gesellschafterdarlehen, die nach niederländischem Recht den regulären progressiven Einkommensteuersätzen unterliegen, in den beiden unteren Progressionsstufen (bis $33.163 €$ ) um drei Prozentpunkte erhöht.

Mitgliedstaaten, die bei der Besteuerung von Anteilseignern im Untersuchungszeitraum 2013 bis 2015 ausschließlich Entlastungen vorgenommen haben, sind Bulgarien, Estland, Malta, Spanien und Zypern. Vergünstigungen für Zinseinkünfte gibt es in Bulgarien und in Estland. Bulgarien hat den erst 2013 eingeführten Steuersatz für Zinseinkünfte aus Bankguthaben von 10\% auf $8 \%$ reduziert. Estland, das generell auf eine weitere Besteuerung von Dividenden auf Anteilseignerebene verzichtet, hat die Besteuerung von Zinseinkünften von 21\% auf 20\% reduziert. Malta, dessen progressiver Tarif auf alle Einkünfte außer Bankzinsen Anwendung findet, hat zunächst in 2014 den Steuersatz für Einkünfte zwischen $28.700 €$ und $60.000 €$ von $32 \%$ auf $29 \%$ gesenkt und diese Progressionsstufe ab 2015 gestrichen. Für Einkünfte in dieser Stufe kommt somit nur noch ein Steuersatz von 25\% zur Anwendung. Spanien hat sehr kurzfristig eine erst für 2016 geplante Steuersenkung mit sofortiger Wirkung zum 12. Juli 2015 eingeführt. Für Kapitaleinkünfte gilt weiterhin ein dreistufiger, progressiver Tarif mit veränderten Steuersätzen und Progressionsstufen. Die obere Grenze der mittleren Progressionsstufe wurde von $24.000 €$ auf $50.000 €$ erhöht. Zugleich wurden die Steuersätze von 21\%, 25\% bzw. 27\% auf 19,5\%, 21,5\% bzw. 23,5\% gesenkt. In Zypern unterliegen Kapitaleinkünfte keiner Einkommensteuer, sondern einer Sonderabgabe (defence contribution). Diese wurde 2014 für Dividenden von 20\% auf 17\% reduziert.

\subsection{Zwischenfazit}

Für Kapitalgesellschaften lässt sich im Untersuchungszeitraum 2013 bis 2015 ein Sinken der tariflichen Steuersätze feststellen, wobei der Trend in Deutschland gegenläufig ist. Die in den Mitgliedstaaten neu eingeführten Regelungen bei der Bemessungsgrundlage orientieren sich insbesondere bei Zinsabzugsbeschränkungen sehr stark an den deutschen Regelungen. Auf Anteilseignerebene ergibt sich dagegen ein differenziertes Bild. In der Mehrzahl der Mitgliedstaaten, die Änderungen durchführen, werden zwar steuerliche Entlastungen für Kapitaleinkünfte vorgenommen. Es lässt sich jedoch nicht erkennen, dass eine bestimmt Finanzierungsform (Eigen- oder Fremdkapital) steuerlich eher bevorzugt wird, da sowohl die Steuersätze für Dividenden und/oder Zinsen geändert werden. 


\section{Quantitative Analyse der Steueränderungen in der Europäischen Union 2013-2015}

\subsection{Methodik: European Tax Analyzer}

Im Rahmen der folgenden quantitativen Untersuchung wird auf das finanzplangestützte Simulationsmodell European Tax Analyzer zurückgegriffen. ${ }^{61}$ Bei dieser Simulation wird über einen Zeitraum von zehn Perioden die effektive Steuerbelastung von Kapitalgesellschaften sowie der Gesamtebene (Kapitalgesellschaft und Anteilseigner) unter Berücksichtigung aller relevanten Steuerarten, Tarife sowie bedeutsamer bilanzieller und steuerlicher Wahlrechte berechnet. Der hohe Detaillierungsgrad der Simulation erlaubt z. B. die Berücksichtigung von verschiedenen Abschreibungsregeln (Immobilien, bewegliches Sachanlagevermögen und immaterielle Wirtschaftsgüter mit jeweiliger Spezifikation der Methode, der Abschreibungssätze und der betriebsgewöhnlichen Nutzungsdauer), Regelungen zur Vorratsbewertung, betrieblichen Altersversorgung, Gesellschafterfremdfinanzierung und der Verlustverrechnung im Rahmen der Körperschaftsteuer. ${ }^{62}$ Auch andere Steuerarten wie Grundsteuer oder Gewerbesteuer in Deutschland können detailliert modelliert werden. Auf Ebene der Anteilseigner können der Familienstand sowie die gesellschafts- und schuldrechtlichen Beziehungen zwischen Gesellschafter und Gesellschaft abgebildet werden.

Als Maßgröße der effektiven Steuerbelastung dient die steuerbedingte Reduktion des Endvermögens auf Unternehmensebene bzw. der Gesamtebene am Ende der zehn Simulationsperioden. Die jährlich anfallenden Steuerzahlungen werden mittels einer Veranlagungssimulation ermittelt. Stille Reserven/stille Lasten, die im Laufe der Simulation bei den bilanzierten Vermögensgegenständen oder Schulden entstehen, werden in der letzten Simulationsperiode berücksichtigt und führen zu Steuerbe- oder -entlastungen. Am Ende der Simulation vorhandene Verlust- oder EBITDA-Vorträge werden mit 50\% (zeitlich und betragsmäßig unbegrenzt) bzw. 25\% (zeitlich oder betragsmäßig begrenzt) bewertet und dem Endvermögen zugeschlagen.

Ausgangsgröße für die Berechnung der periodischen Steuerzahlungen bilden Daten der Vermögens- und Kapitalausstattung sowie eines detaillierten Unternehmensplans einer repräsentativen großen europäischen Kapitalgesellschaft. ${ }^{63}$ Die Musterunternehmen wurden aus der Bilanzdatenbank AMADEUS (Update September 2013) generiert. ${ }^{64}$ Die daraus gewonnene Datenbasis besteht aus vollständigen Bilanzen, Gewinn- und Verlustrechnungen sowie Personalangaben von 2.424.612 Unternehmen für das Jahr 2011 (da-

\footnotetext{
61 Vgl Jacobs, O. H./Spengel, C., European Tax Analyzer, 1996; Spengel, C./Zinn, B., StuW 2011, S. 177; Spengel, C. et al., SME Taxation in Europe, 2015, S. 69-73.

${ }^{62}$ Für weitere Vorschriften, die berücksichtigt werden, vgl. Spengel, C./Zinn, B., StuW 2011, S. 177.

${ }^{63}$ Die Einteilung nach Größenklassen richtet sich nach der Empfehlung der EU-Kommission vom 6. Mai 2003.

${ }^{64}$ Das Update September 2013 der Bilanzdatenbank AMADEUS enthält die Bilanzdaten für über 14 Mio. europäische Unternehmen im betrachteten Finanzjahr 2011.
} 
von 25.490 große Unternehmen). ${ }^{65}$ Aus den Daten der einzelnen Kapitalgesellschaften wurden die Bilanz- und Ertragskennzahlen zunächst branchen- und länderspezifisch gemittelt und anschließend zu einem ungewichteten EU-28 Durchschnitt aggregiert.66 Abbildung 1 zeigt die resultierende Bilanz sowie bedeutende Bilanz- und Erfolgskennzahlen des Modellunternehmens in der Mitte des Betrachtungszeitraums (Periode 6 von 10). ${ }^{67}$

\section{Abbildung 1: Steuerbilanz und Erfolgs-/Bilanzkennzahlen des Modellunter- nehmens (Periode 6 von 10)}

\section{Steuerbilanz}

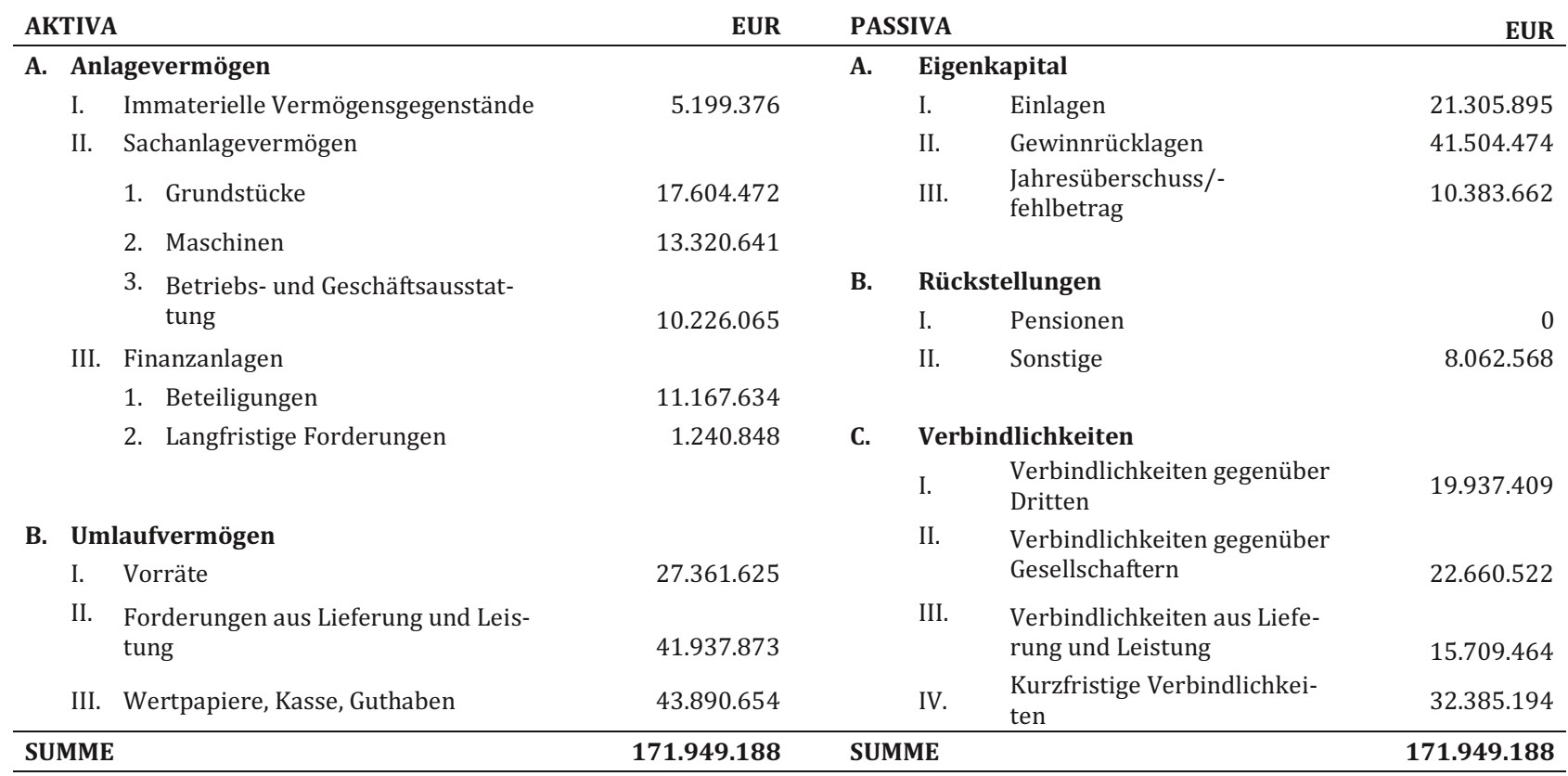

\section{Erfolgs- und Bilanzkennzahlen}

\begin{tabular}{|c|c|c|c|c|c|}
\hline Anlagenintensität & $\begin{array}{c}\text { Umsatzrentabili- } \\
\text { tät nach Steuern }\end{array}$ & $\begin{array}{c}\text { Eigenkapital- } \\
\text { rentabilität }\end{array}$ & $\begin{array}{c}\text { Eigenkapital- } \\
\text { quote }\end{array}$ & Vorratsintensität & Personalintensität \\
\hline $23,93 \%$ & $4,95 \%$ & $16,53 \%$ & $42,57 \%$ & $15,91 \%$ & $11,42 \%$ \\
\hline
\end{tabular}

Die Entwicklung des Unternehmens während der 10-jährigen Simulationsperiode wird durch einen detaillierten Unternehmensplan bestimmt. Dieser enthält Annahmen über die Produktion und den Absatz sowie bezüglich des Investitions-, Finanzierungs- und Ausschüttungsverhaltens des Unternehmens. Für die gesamtwirtschaftlichen Daten

\footnotetext{
${ }^{65}$ Ausgeschlossen wurden Daten von nicht betrachteten Rechtsformen (Personengesellschaften, Einzelunternehmen) oder bestimmten Branchen (z. B. Kreditinstitute) sowie Unternehmen, bei denen ausschließlich ein Konzernabschluss vorlag. Einige Firmendaten sind zudem nur unvollständig vorhanden und wurden für eine weitere Betrachtung ausgeschlossen.

${ }^{66}$ Für eine detaillierte Beschreibung siehe ausführlich Spengel, C.et al., SME Taxation in Europe, 2015, Annex 1, S. 58-70.

${ }^{67}$ Die betriebliche Altersversorgung wird in der Simulation im Ausgangsfall über jährliche Beiträge an eine Pensionskasse abgebildet, weshalb die Bilanzposition Null beträgt.
} 
(Preissteigerungsraten, Zinssätze) wurden folgende Werte aus Veröffentlichungen von Eurostat, des Statistischen Bundesamts und der Europäischen Zentralbank ermittelt: ${ }^{68}$

- Preissteigerungsraten: 2,7\% für allgemeine Lebenshaltungskosten, 2,5\% für Grundstoffe, 2,2\% für Personalkosten und 2,7\% für Investitionsgüter;

- Soll- und Habenzinssätze: 1,1\% bzw. 2,5\% für kurz- bzw. langfristige Forderungen und 3,9\% bzw. 3,5\% für kurz- bzw. langfristige Verbindlichkeiten.

Die Anteilseignerebene wird durch zehn natürliche Personen mit Beteiligungsquoten zwischen 5\% und 51\% repräsentiert. Neben einer jährlichen Dividendenzahlung erhalten die Anteilseigner Zinsen aus Gesellschafterdarlehen (Zinssatz: 3,5\%). Zur Berechnung und Darstellung von Progressionseffekten wird für jeden Anteilseigner zudem ein virtuelles Einkommen i.H.v. $50.000 €$ unterstellt. Die Steuer, die auf diesem virtuellen Einkommen lastet, wird am Ende der jeweiligen Steuerberechnung von der Gesamtsteuerbelastung abgezogen. 69

\subsection{Entwicklung der effektiven Steuerbelastung im Zeitraum 2013-2015}

Tabelle 3 zeigt die effektive Steuerbelastung des Modellunternehmens nach 10 Simulationsperioden für die Jahre 2013 bis 2015 auf Ebene der Kapitalgesellschaft und der Gesamtebene (aggregierte Steuerbelastung von Unternehmens- und Anteilseignerebene). Die Anordnung der Länder richtet sich dabei nach der Höhe der effektiven Steuerbelastung für das Jahr 2015.

\subsubsection{Ebene der Kapitalgesellschaft}

Bei Betrachtung der effektiven Steuerbelastung auf Kapitalgesellschaftsebene finden sich in der Spitzengruppe (Bulgarien bis Polen) nach wie vor überwiegend osteuropäische Mitgliedstaaten. Es fällt auf, dass die Platzierungen der Länder im Zeitablauf sehr konstant bleiben. Einzig Lettland verliert durch die Abschaffung des fiktiven Zinsabzugs auf Eigenkapital im Jahr 2014 einen Rang und die effektive Steuerbelastung steigt um 6,35\% auf 27,55 Mio. €. Die Verminderung der Effektivbelastung um 2,17\% in Irland, das durchweg Platz 2 belegt, lässt sich mit einer erheblichen Reduzierung der dortigen Grundsteuersätze begründen.

Eine weitaus höhere Dynamik ist im Mittelfeld (Estland bis Griechenland) festzustellen. Abgesehen von Schweden ändert sich in allen Ländern dieser Gruppe die effektive Steuerbelastung. Durch die Reduzierung des KSt-Satzes in Finnland in 2014 verringert sich die effektive Steuerbelastung um 17,38\%. Großbritannien und Dänemark verbessern sich von 2013 bis 2015 infolge der verminderten KSt-Sätze jeweils um zwei Ränge. Portugal und Griechenland haben die Plätze getauscht und Griechenland hat sich mit einer effektiven Steuerbelastung von 49,63 Mio. € den Steuerbelastungen der Schlussgruppe angenähert.

\footnotetext{
${ }^{68}$ Alle Preissteigerungsraten und Zinssätze beziehen sich auf das Jahr 2012.

${ }^{69}$ Siehe ausführlich: Spengel, C./Zinn, B., StuW 2011, S. 179.
} 
Tabelle 3: Effektive Steuerbelastungen 2013 bis 2015 (Unternehmens- und Gesamtebene nach 10 Perioden) in Mio. $€$ und jährliche Veränderungen in \%

\begin{tabular}{|c|c|c|c|c|c|c|c|c|}
\hline \multicolumn{9}{|c|}{ Besteuerung auf Ebene der Kapitalgesellschaft } \\
\hline \multirow[b]{2}{*}{ Land } & \multicolumn{2}{|c|}{2013} & \multicolumn{2}{|l|}{2014} & \multicolumn{2}{|l|}{2015} & \multicolumn{2}{|c|}{$\Delta$ Belastung } \\
\hline & $\begin{array}{l}\text { Belastung in } \\
\text { Mio. } €\end{array}$ & Rang & $\begin{array}{l}\text { Belastung in } \\
\text { Mio. } €\end{array}$ & Rang & $\begin{array}{c}\text { Belastung in } \\
\text { Mio. } €\end{array}$ & Rang & $\begin{array}{c}2014 \mathrm{zu} \\
2013 \\
\end{array}$ & $\begin{array}{c}2015 \mathrm{zu} \\
2014\end{array}$ \\
\hline Bulgarien & 17,00 & 1 & 17,00 & 1 & 17,00 & 1 & $0,00 \%$ & $0,00 \%$ \\
\hline Irland & 22,01 & 2 & 21,53 & 2 & 21,53 & 2 & $-2,17 \%$ & $-0,01 \%$ \\
\hline Zypern & 24,96 & 3 & 24,96 & 3 & 24,60 & 3 & $0,00 \%$ & $-1,47 \%$ \\
\hline Rumänien & 27,39 & 5 & 27,39 & 4 & 27,39 & 4 & $0,00 \%$ & $0,00 \%$ \\
\hline Lettland & 25,91 & 4 & 27,55 & 5 & 27,55 & 5 & $6,35 \%$ & $0,00 \%$ \\
\hline Slowenien & 27,95 & 6 & 27,95 & 6 & 27,95 & 6 & $0,00 \%$ & $0,00 \%$ \\
\hline Litauen & 28,23 & 7 & 28,23 & 7 & 28,23 & 7 & $0,00 \%$ & $0,00 \%$ \\
\hline Tschech. Rep. & 31,54 & 8 & 31,52 & 8 & 31,52 & 8 & $-0,05 \%$ & $0,00 \%$ \\
\hline Kroatien & 32,46 & 9 & 32,46 & 9 & 32,46 & 9 & $0,00 \%$ & $0,00 \%$ \\
\hline Polen & 32,88 & 10 & 32,90 & 10 & 32,92 & 10 & $0,06 \%$ & $0,08 \%$ \\
\hline Estland & 34,57 & 11 & 34,57 & 12 & 32,96 & 11 & $0,00 \%$ & $-4,65 \%$ \\
\hline Finnland & 41,37 & 14 & 34,18 & 11 & 34,49 & 12 & $-17,38 \%$ & $0,92 \%$ \\
\hline Schweden & 36,87 & 12 & 36,87 & 13 & 36,87 & 13 & $0,00 \%$ & $0,00 \%$ \\
\hline Großbritannien & 41,58 & 16 & 38,43 & 15 & 36,94 & 14 & $-7,58 \%$ & $-3,87 \%$ \\
\hline Slowakei & 39,13 & 13 & 37,53 & 14 & 37,58 & 15 & $-4,09 \%$ & $0,13 \%$ \\
\hline Dänemark & 43,73 & 18 & 42,94 & 17 & 41,36 & 16 & $-1,81 \%$ & $-3,68 \%$ \\
\hline Niederlande & 41,52 & 15 & 41,54 & 16 & 41,48 & 17 & $0,06 \%$ & $-0,14 \%$ \\
\hline Portugal & 49,87 & 19 & 46,78 & 19 & 43,56 & 18 & $-6,19 \%$ & $-6,88 \%$ \\
\hline Griechenland & 43,57 & 17 & 45,13 & 18 & 49,63 & 19 & $3,56 \%$ & $9,98 \%$ \\
\hline Luxemburg & 50,95 & 20 & 50,95 & 20 & 50,95 & 20 & $0,00 \%$ & $0,00 \%$ \\
\hline Österreich & 51,09 & 21 & 51,09 & 21 & 51,09 & 21 & $0,00 \%$ & $0,00 \%$ \\
\hline Italien & 56,18 & 24 & 55,82 & 24 & 52,46 & 22 & $-0,65 \%$ & $-6,02 \%$ \\
\hline Deutschland & 53,38 & 22 & 53,50 & 22 & 53,58 & 23 & $0,23 \%$ & $0,15 \%$ \\
\hline Belgien & 54,15 & 23 & 54,15 & 23 & 54,15 & 24 & $0,00 \%$ & $0,00 \%$ \\
\hline Spanien & 58,13 & 26 & 58,13 & 26 & 55,31 & 25 & $0,00 \%$ & $-4,84 \%$ \\
\hline Malta & 57,45 & 25 & 57,45 & 25 & 57,45 & 26 & $0,00 \%$ & $0,00 \%$ \\
\hline Ungarn & 62,82 & 27 & 62,83 & 27 & 63,67 & 27 & $0,01 \%$ & $1,34 \%$ \\
\hline Frankreich & 78,96 & 28 & 75,94 & 28 & 75,91 & 28 & $-3,82 \%$ & $-0,05 \%$ \\
\hline
\end{tabular}

\begin{tabular}{|lcccccc|cc|}
\hline \multicolumn{7}{c}{ Besteuerung der Gesamtebene (Unternehmens- und Anteilseignerebene) } \\
\hline \multicolumn{1}{c}{ Land } & $\mathbf{2 0 1 3}$ & \multicolumn{2}{c}{$\mathbf{2 0 1 4}$} & \multicolumn{2015}{c|}{$\mathbf{2 0 1 5}$} & \multicolumn{2}{c|}{ Belastung } \\
\hline Belastung in & Mio. & Rang & $\begin{array}{c}\text { Belastung in } \\
\text { Mio. }\end{array}$ & Rang & $\begin{array}{c}\text { Belastung in } \\
\text { Mio. }\end{array}$ & Rang & 2014 zu & 2015 zu \\
\hline Bulgarien & 25,43 & 1 & 25,39 & 1 & 25,39 & 1 & $-0,14 \%$ & $0,00 \%$ \\
Estland & 36,32 & 2 & 36,32 & 2 & 34,63 & 2 & $0,00 \%$ & $-4,65 \%$ \\
Slowakei & 41,55 & 4 & 39,95 & 3 & 40,00 & 3 & $-3,86 \%$ & $0,12 \%$ \\
Lettland & 40,86 & 3 & 42,35 & 4 & 42,35 & 4 & $3,63 \%$ & $0,00 \%$ \\
Litauen & 55,68 & 8 & 50,31 & 5 & 50,31 & 5 & $-9,65 \%$ & $0,00 \%$ \\
Rumänien & 51,06 & 5 & 51,06 & 6 & 51,06 & 6 & $0,00 \%$ & $0,00 \%$ \\
Zypern & 56,00 & 9 & 51,80 & 7 & 51,49 & 7 & $-7,50 \%$ & $-0,59 \%$ \\
Kroatien & 55,42 & 7 & 55,42 & 9 & 52,69 & 8 & $0,00 \%$ & $-4,93 \%$ \\
Tschech. Rep. & 53,11 & 6 & 53,10 & 8 & 53,10 & 9 & $-0,03 \%$ & $0,00 \%$ \\
Polen & 59,94 & 10 & 59,95 & 10 & 59,97 & 10 & $0,02 \%$ & $0,03 \%$ \\
Malta & 60,61 & 11 & 60,60 & 11 & 60,59 & 11 & $-0,02 \%$ & $-0,02 \%$ \\
Slowenien & 64,74 & 12 & 64,74 & 12 & 64,74 & 12 & $0,00 \%$ & $0,00 \%$ \\
Ungarn & 64,88 & 13 & 64,88 & 13 & 65,73 & 13 & $0,01 \%$ & $1,30 \%$ \\
Griechenland & 82,99 & 17 & 66,09 & 14 & 74,28 & 14 & $-20,37 \%$ & $12,40 \%$ \\
Schweden & 78,33 & 14 & 78,33 & 16 & 78,33 & 15 & $0,00 \%$ & $0,00 \%$ \\
Niederlande & 78,54 & 15 & 78,01 & 15 & 78,47 & 16 & $-0,68 \%$ & $0,59 \%$ \\
Luxemburg & 79,10 & 16 & 79,10 & 17 & 79,72 & 17 & $0,00 \%$ & $0,78 \%$ \\
Großbritannien & 83,51 & 18 & 81,32 & 18 & 80,22 & 18 & $-2,63 \%$ & $-1,35 \%$ \\
Portugal & 84,94 & 22 & 82,72 & 20 & 80,40 & 19 & $-2,62 \%$ & $-2,80 \%$ \\
Finnland & 85,05 & 23 & 81,40 & 19 & 81,69 & 20 & $-4,29 \%$ & $0,35 \%$ \\
Österreich & 84,10 & 20 & 84,10 & 21 & 84,10 & 21 & $0,00 \%$ & $0,00 \%$ \\
Belgien & 84,22 & 21 & 84,27 & 22 & 84,27 & 22 & $0,06 \%$ & $0,00 \%$ \\
Italien & 83,83 & 19 & 86,84 & 23 & 84,31 & 23 & $3,59 \%$ & $-2,92 \%$ \\
Deutschland & 88,41 & 24 & 88,50 & 24 & 88,55 & 24 & $0,10 \%$ & $0,06 \%$ \\
Dänemark & 98,95 & 25 & 98,38 & 25 & 97,40 & 25 & $-0,57 \%$ & $-1,01 \%$ \\
Irland & 99,58 & 26 & 99,42 & 26 & 99,37 & 26 & $-0,16 \%$ & $-0,05 \%$ \\
Spanien & 106,56 & 27 & 106,56 & 27 & 101,56 & 27 & $0,00 \%$ & $-4,69 \%$ \\
Frankreich & 122,11 & 28 & 120,31 & 28 & 120,29 & 28 & $-1,47 \%$ & $-0,02 \%$ \\
\hline
\end{tabular}


Diese Schlussgruppe (Luxemburg bis Frankreich) ist von einer erheblich geringeren Dynamik im Vergleich zum Mittelfeld geprägt. Die Platzierungen der Länder bleiben weitgehend unverändert. Die Abzugsfähigkeit der vollen Lohnkosten bei der italienischen Wertschöpfungssteuer IRAP seit 2015 reduziert die effektive Steuerbelastung um 4,84\%, wodurch Italien Deutschland auf Rang 23 verdrängt. Spanien verbessert sich durch die Reduzierung des KSt-Satzes von 30\% auf 28\% auf Platz 25 und nähert sich den davor liegenden Mitgliedstaaten stark an. Die eingeführte Steuergutschrift für Lohnkosten in Frankreich senkt zwar die effektive Steuerbelastung, dennoch bleibt Frankreich mit großem Abstand Schlusslicht im Länderranking.

Neben der Gesamtbetrachtung liefert ein fokussierter Vergleich einzelner Ländergruppen mit Deutschland aufschlussreiche Erkenntnisse hinsichtlich der Position Deutschlands im EU-Steuerwettbewerb. Abbildung 2 zeigt die effektive Steuerbelastung auf Ebene der Kapitalgesellschaft für die acht Mitgliedstaaten mit dem höchsten Bruttoinlandsprodukt (BIP) im Jahr 2014.70

\section{Abbildung 2: Effektive Steuerbelastung (Ebene der Kapitalgesellschaft) nach 10 Simulationsperioden 2013-2015 für die acht EU-Mitgliedstaaten mit dem höchsten BIP}

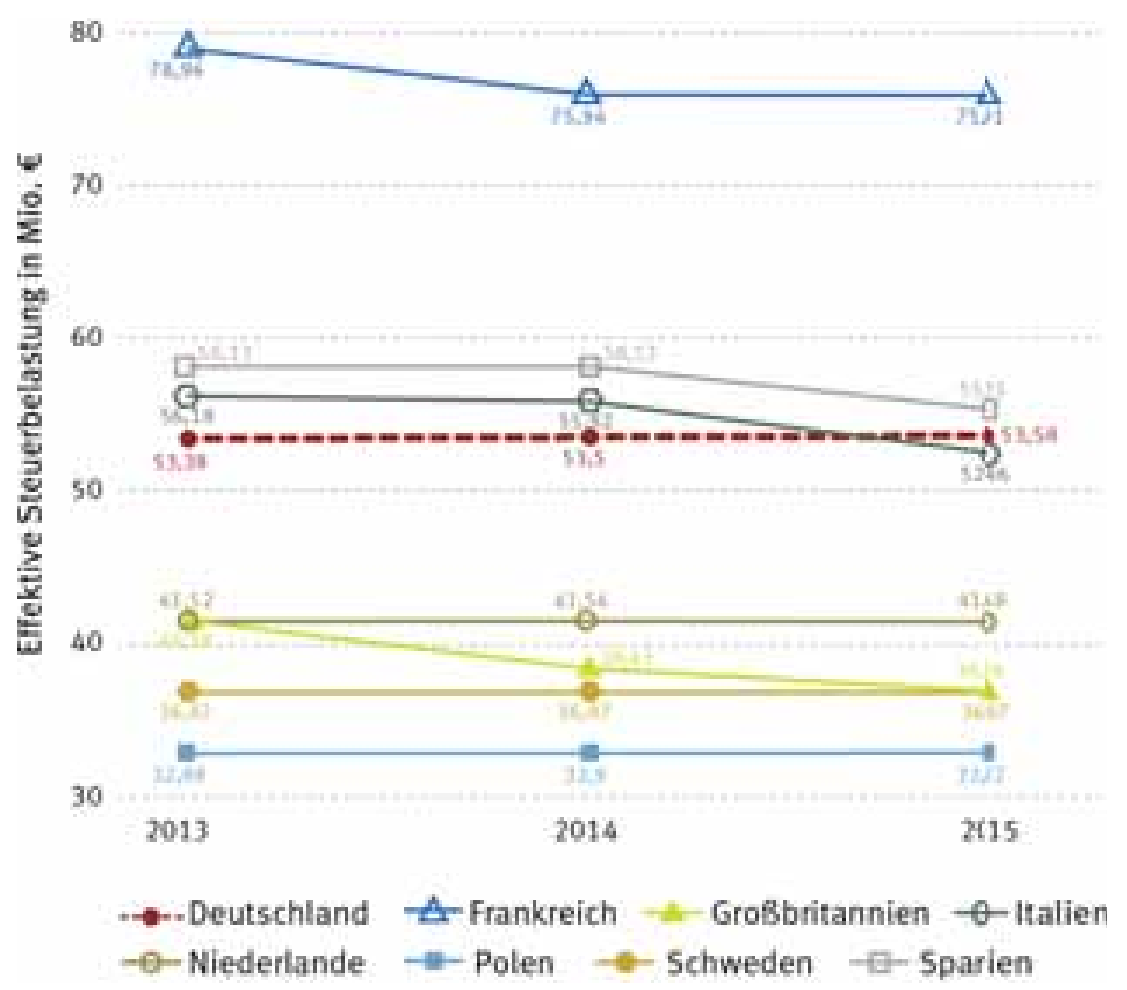

Zu Polen, Schweden und den Niederlanden besteht hinsichtlich der effektiven Steuerbelastung ein unverändert großer Abstand. Durch die jährlichen Steuersatzsenkungen in Großbritannien erhöht sich der Unterschied der effektiven Steuerbelastungen zwischen

70 Vgl. Eurostat, Bruttoinlandsprodukt, 2015. 
beiden Ländern von 22,10\% im Jahr 2013 auf nun 31,18\%, sodass Deutschland immer weiter hinter Großbritannien zurückfällt. Auch in Italien lässt sich eine Steigerung der steuerlichen Standortattraktivität durch die Veränderung der Bemessungsgrundlage für die IRAP feststellen. Dies führt dazu, dass die effektive Steuerbelastung in Italien nun mit 52,46 Mio. € geringer ist als in Deutschland (53,58 Mio. €). Spanien hat sich Deutschland durch die Senkung des KSt-Satzes erheblich angenähert. Durch die erneute Senkung des KSt-Satzes ab 2016 auf 25\% ist davon auszugehen, dass die effektive Steuerbelastung in Spanien zukünftig geringer als in Deutschland sein wird. Innerhalb des Vergleichs mit wirtschaftlich ähnlich starken Ländern fällt Deutschland bei Betrachtung der effektiven Steuerbelastung auf Unternehmensebene somit zurück.

\subsubsection{Gesamtebene (Unternehmens- und Anteilseignerebene)}

Wie Tabelle $3 \mathrm{zu}$ entnehmen ist, ergeben sich bei der zusätzlichen Einbeziehung der Anteilseignerebene für einzelne Mitgliedstaaten erhebliche Veränderungen in der Platzierung. Während Irland von dem zweiten auf den 26. Rang zurückfällt, verbessern sich Länder wie Ungarn oder die Slowakische Republik erheblich. Diese Rangverschiebungen zeigen die Bedeutung der Einbeziehung der Anteilseignerebene. Irland besteuert z.B. Dividendeneinkünfte bereits ab $42.800 €$ mit einem Steuersatz von $40 \%$ zuzüglich einer progressiv ausgestalteten Zuschlagsteuer. Estland und die Slowakische Republik verzichten dagegen vollständig auf eine Besteuerung der Dividenden beim Anteilseigner und besteuern Zinseinkünfte mit moderaten Steuersätzen von ca. 20\%.

In der Spitzengruppe (Bulgarien bis Ungarn) bleibt die Rangfolge der Länder weitgehend stabil. Nur Litauen und Zypern können sich maßgeblich durch die Reduktion des Steuersatzes für Dividendeneinkünfte im Jahr 2014 um drei bzw. zwei Plätze verbessern, sodass die Gesamtsteuerbelastung auf 50,31 bzw. 51,49 Mio. € sinkt. Die Abnahme der Effektivbelastung in Kroatien im Jahr 2015 lässt sich mit der erheblichen Reduktion des Steuersatzes für Zinseinkünfte von $40 \%$ auf $12 \%$ begründen.

Das Mittelfeld beginnt bei Griechenland, das mit einer Gesamtsteuerbelastung von 74,28 Mio. € für das Jahr 2015 einen großen Unterschied zu Ungarn (65,73 Mio. €) aufweist. Während sich im Jahr 2014 die Gesamtsteuerbelastung durch die erhebliche Reduzierung des Steuersatzes von Dividendeneinkünfte von 25\% auf 10\% sehr stark verringert hat, lässt sich die Erhöhung im Jahr 2015 auf den gestiegenen KSt-Satz (29\% statt 26\%) und die Erhöhung einer Sonderabgabe auf Anteilseignerebene zurückführen. Insgesamt verbessert sich Griechenland dennoch um drei Ränge. Die weiteren kleineren Änderungen im Mittelfeld (Griechenland bis Finnland) lassen sich hauptsächlich auf Veränderungen bei der Besteuerung auf der Gesellschaftsebene zurückführen. In Finnland wird der Effekt der Senkung des KSt-Satz in 2014, der die effektive Steuerbelastung auf Ebene der Kapitalgesellschaft um 17,36\% reduziert, durch die Erhöhung der Anteilseignerbesteuerung abgeschwächt. Die Gesamtentlastung beträgt lediglich 4,29\%. 
Die bei Österreich beginnende Schlussgruppe verzeichnet sehr wenige Rangänderungen. Einzig Italien fällt durch die Erhöhung der Steuersätze für Dividenden und Zinsen von 20\% auf 26\% im Jahr 2014 von Rang 19 auf Rang 23 zurück. Ab Platz 24 (Deutschland) bleibt das Gesamtranking trotz einiger Entlastungen wie z.B. in Spanien vollständig stabil. Spanien konnte durch die vorgenommenen Reformen jedoch den Abstand zu Dänemark und Irland erheblich reduzieren.

Wie bei der Ebene der Kapitalgesellschaft soll auch bei der Gesamtebene ein Vergleich der Position Deutschlands mit anderen wirtschaftsstarken Mitgliedstaaten erfolgen. Der Vergleich der Gesamtsteuerbelastung innerhalb dieser Ländergruppe ist insbesondere für inhabergeführte, mittelständische Unternehmen relevant. Nur durch die Einbeziehung beider Ebenen (Unternehmen und Anteilseigner) lässt sich beurteilen, inwieweit die Wettbewerbsfähigkeit deutscher mittelständischer Unternehmen durch nachteilige steuerliche Bedingungen im Vergleich zu ähnlich strukturierten Unternehmen in anderen europäischen Ländern beeinträchtigt wird. Abbildung 3 zeigt die effektive Steuerbelastung für die Gesamtebene für die acht Mitgliedstaaten mit dem höchsten BIP im Jahr 2014.71

\section{Abbildung 3: Effektive Gesamtsteuerbelastung (Unternehmens- und Anteilseignerebene) nach 10 Simulationsperioden 2013-2015 für die acht EU- Mitgliedstaaten mit dem höchsten BIP}

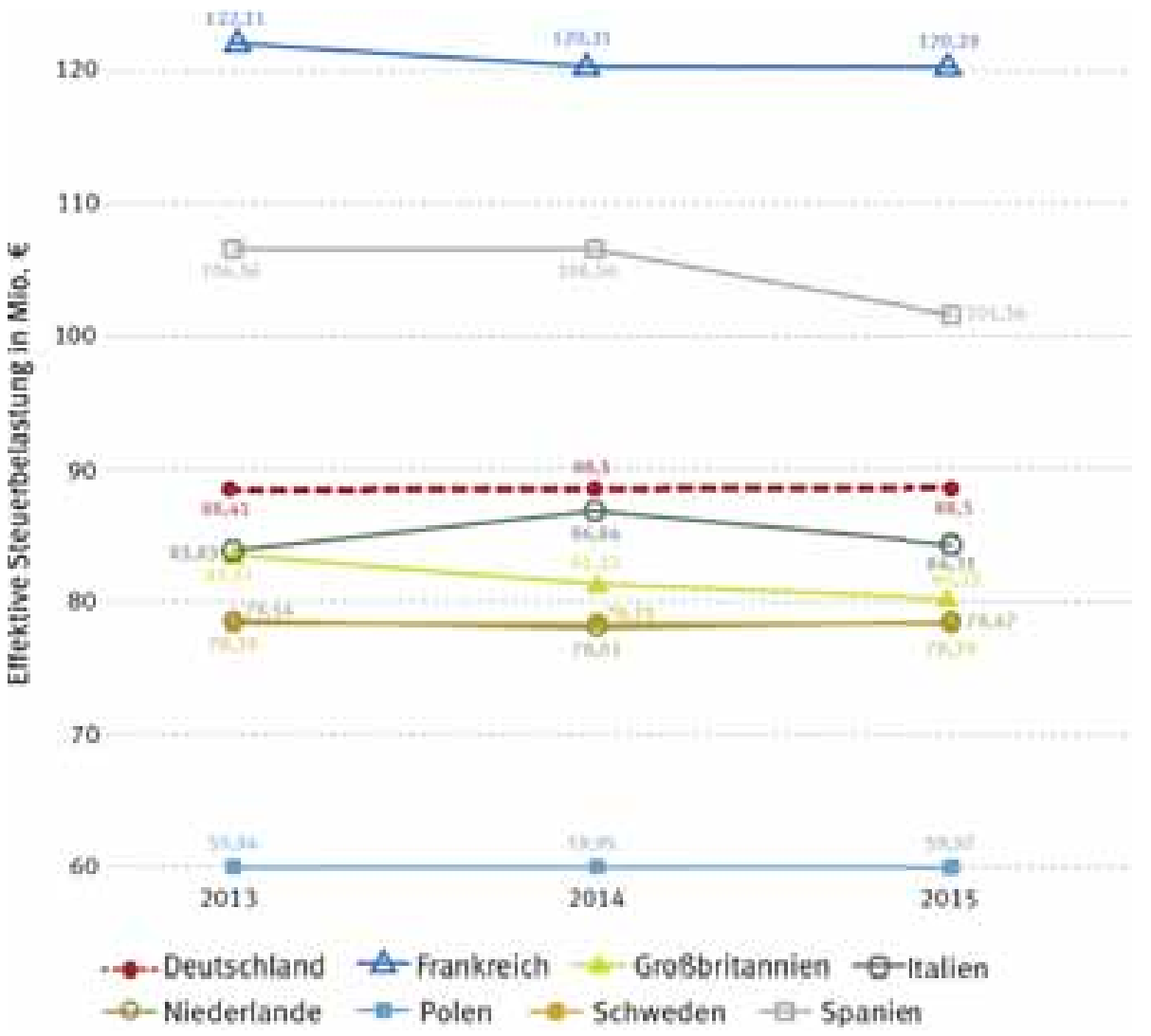

71 Vgl. Eurostat, Bruttoinlandsprodukt, 2015. 
Auch bei Betrachtung der Gesamtebene ergibt sich ein Rückstand Deutschlands auf Polen, Schweden und die Niederlande. Es ist jedoch auffällig, dass der Abstand hier nicht so groß ist wie bei der alleinigen Betrachtung der Ebene der Kapitalgesellschaft. Dies deutet daraufhin, dass diese Länder die niedrige effektive Steuerbelastung auf Ebene der Kapitalgesellschaft durch eine tendenziell höhere Besteuerung der Anteilseigner ausgleichen. Italien liegt im Gegensatz zur Unternehmensebene in allen drei Jahren vor Deutschland, während zu Spanien immer noch ein sehr großer Abstand besteht. Grund dafür ist insbesondere das Beibehalten einer Vermögensteuer in Spanien, die nach einer Abschaffung im Jahr 2008 bereits seit 2011 wieder erhoben wird. Insgesamt betrachtet fällt Deutschland im Vergleich mit wichtigen Wettbewerbsländern zwar etwas zurück, die Unterschiede in der effektiven steuerlichen Gesamtbelastung von Unternehmensund Anteilseignerebene sind hier jedoch nicht so groß wie bei der alleinigen Betrachtung der Unternehmensebene.

\section{Thesenförmige Zusammenfassung}

1. In vielen Bereichen des deutschen Steuersystems gibt es derzeit konzeptionelle Mängel, deren Beseitigung nicht proaktiv, sondern erst durch Impulse der Rechtsprechung von BVerfG und BFH angegangen wird.

2. Die derzeit geplante Umsetzung der von der Rechtsprechung geforderten Änderungen bei einzelnen Steuerarten (ErbSt, GrESt, GrSt) lässt eine weitere Komplizierung des deutschen Steuersystems befürchten und widerspricht den Zielen des Koalitionsvertrages. Diese Konsequenzen sind auch bei anderen steuerlichen Gesetzesinitiativen, wie der geplanten Steuerpflicht für Veräußerungsgewinne aus Streubesitz, zu erwarten.

3. Nahezu für alle Steuerarten lässt sich in Deutschland eine Erhöhung der tariflichen Steuerbelastung in den letzten Jahren und auch in dieser Legislaturperiode feststellen. Neben der GrESt und GrSt betrifft dies insbesondere die GewSt. Dies führt neben einer zunehmenden Belastung gewerblicher Einkünfte im Rahmen der Einkommensteuer auch zu einer kontinuierlich steigenden effektiven Tarifbelastung bei Kapitalgesellschaften.

4. Die Entwicklung in Deutschland läuft dem derzeitigen Trend in der Europäischen Union diametral entgegen, da dort mehrere Mitgliedstaaten ihre Körperschaftsteuersätze im Zeitraum 2013 bis 2015 teilweise erheblich gesenkt haben.

5. Bei der Verbreiterung der Bemessungsgrundlage der Körperschaftsteuer orientieren sich viele Mitgliedstaaten an den derzeitigen Regelungen in Deutschland. Dies gilt vor allem für neue Regelungen zur Beschränkung des Zinsausgabenabzugs. Verlustabzugsmöglichkeiten werden in zeitlicher und betragsmäßiger Hinsicht in mehreren Mitgliedsstaaten erheblich eingeschränkt.

6. Kein eindeutiger Trend lässt sich bei der Besteuerung der Anteilseigner erkennen. Dies gilt sowohl für die generelle tarifliche Steuerbelastung wie auch für die 
unterschiedliche steuerliche Behandlung einzelner Einkunftsarten wie Zinsen und Dividenden.

7. In einem quantitativen Vergleich der effektiven Steuerbelastungen zwischen Kapitalgesellschaften in der Europäischen Union gibt es bei der alleinigen Betrachtung der Unternehmensebene insbesondere bei Ländern mit einer mittleren Platzierung viele Veränderungen. Mit Finnland und Portugal sind in dieser Gruppe auch die Länder mit den höchsten Entlastungen (-16,46\% bzw. -13,07\%) im Untersuchungszeitraum 2013 bis 2015 zu finden.

8. Das Ranking der effektiven Gesamtbelastung (Unternehmens- und Anteilseignerebene) bleibt abgesehen von Veränderungen bei einzelnen Mitgliedstaaten sehr stabil. In Litauen und Zypern sinkt die Gesamtbelastung mit 9,65\% bzw. 8,09\% am stärksten, während sich kleinere Zusatzbelastungen nur vereinzelt (z.B. Luxemburg, Ungarn) feststellen lassen.

9. Ein Vergleich Deutschlands mit anderen wirtschaftlich starken Mitgliedstaaten der Europäischen Union offenbart die Konsequenzen des derzeitigen steuerlichen Stillstands. Insbesondere bei der alleinigen Betrachtung der Unternehmensebene vergrößert sich der Abstand zu wichtigen Wettbewerbsländern wie Großbritannien. Für Italien lässt sich für das Jahr 2015 bereits eine geringere effektive Steuerbelastung im Vergleich zu Deutschland feststellen. Da auch Spanien Deutschland ab 2016 voraussichtlich überholen wird, wird Deutschland zukünftig auf Rang 24 der 28 EU-Mitgliedstaaten zurückfallen und die steuerliche Standortattraktivität Deutschlands wird weiter abnehmen.

10. Eine analoge Betrachtung der steuerlichen Gesamtbelastung ist insbesondere für die Beurteilung der Wettbewerbsfähigkeit deutscher mittelständischer Unternehmen von Bedeutung. Hier ergeben sich bisher noch keine gravierenden Konsequenzen, da die Senkung der Steuerbelastung auf Unternehmensebene z.B. in Italien durch eine Erhöhung der Anteilseignerbesteuerung ausgeglichen wird. Deutschland belegt im Ranking daher unverändert Platz 24. 


\section{Literaturverzeichnis}

Andrae, K. (ifst-Schrift 504, 2015): Grundsteuer und Gewerbesteuer: Update 2014, ifstSchrift 504, Berlin 2015

Becker, J. (BB 2011): Grundsteuerrefommodelle im Vergleich - Konzeption und Praxisfolgen, in: BB 2011, S. 535-539

Benz, S./Jetter, J. (DStR 2013): Die Neuregelung zur Steuerpflicht von Streubesitzdividenden, in: DStR 2013, S. 489-496

BFH (Entscheidungsvorschau, 2015): Entscheidungsvorschau, 2015, http://www.bundesfinanzhof.de/print/anhaengigeverfahren/entscheidungsvorschau, Zugriffsdatum: 26.07.2015

BFH, Urteil vom 30.06.2010, II R 60/08, BStBl. 2010 II, S. 897

BFH, Beschluss vom 27.09.2012, II R 9/11, BStBl. 2012 II, S. 899

BFH, Beschluss vom 16.10.2012, I B 128/12, BStBl. 2013 II, S. 30

BFH, Beschluss vom 18.12.2013, I B 85/13, BStBl. 2014 II, S. 947

BFH, Beschluss vom 22.10.2014, II R 16/13, BStBl. 2014 II, S. 957

BFH, Urteil vom 04.06.2014, I R 70/2, BStBl. 2015 II, S. 289

BMF (Investmentbesteuerung, 2015): Entwurf eines Gesetzes zur Reform der Investmentbesteuerung, 2015, http://www.bundesfinanzministerium.de/Web/DE/Themen/Steuern/Steuerarte

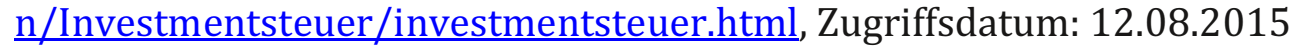

BMF (Neuregelung - Eckwerte, 2015): Neuregelung der Erbschaftsteuer für Unternehmensvermögen - Eckwerte, 2015

BR-Drucksache 201/12, 11.05.2012

BR-Drucksache 281/15, 10.07.2015

BR-Drucksache 291/14, 11.07.2014

BT-Drucksache 16/4841, 23.03.2007

BT-Drucksache 18/5244, 17.06.2015

BT-Drucksache 18/6094, 25.09.2015

Bundesregierung (Protokollerklärung, 2014): Protokollerklärung der Bundesregierung zu einer künftigen Besteuerung von Veräußerungsgewinnen aus Streubesitz, 2014 
Bundesregierung (Gesetzentwurf, 2015): Entwurf eines Gesetzes zur Anpassung des Erbschaftsteuer- und Schenkungsteuergesetzes an die Rechtsprechung des Bundesverfassungsgerichts, 2015, http://www.bundesfinanzministerium.de/Content/DE/Downloads/Gesetze/201 5-07-08-G-z-Anpassung-d-ErbStR-u-SchenkSt-a-d-Rspr-dBVerfG.pdf? blob=publicationFile\&v=2, Zugriffsdatum: 04.08.2015

BVerfG (Jahresvorschau, 2015): Jahresvorschau 2015, 2015, http://www.bundesverfassungsgericht.de/DE/Verfahren/Jahresvorausschau/vs 2015/vorausschau 2015 node.html, Zugriffsdatum: 10.08.2015

BVerfG, Beschluss vom 07.11.2006, 1 BvL 10/02, BGBl. 2007 I, S. 194

BVerfG, Beschluss vom 23.06.2015, 1 BvL 13/11, BGBl. 2015 I, S. 1423

BVerfG, Urteil vom 17.12.2014, 1 BvL 21/12, BStBl. 2015 II, S. 50

CDU (Kalte Progression, 2014): Wichtigstes Ziel: Haushalt ohne neue Schulden, 2014, https://www.cdu.de/artikel/wichtigstes-ziel-haushalt-ohne-neue-schulden, Zugriffsdatum: 01.08.2015

CDU/CSU und FDP (Koalitionsvertrag, 2009): Wachstum. Bildung. Zusammenhalt., 2009, http://www.csu.de/common/ migrated/csucontent/091026 koalitionsvertrag.p df, Zugriffsdatum: 10.07.2015

CDU/CSU und SPD (Koalitionsvertrag, 2005): Gemeinsam für Deutschland - mit Mut und Menschlichkeit, 2005, https://www.cdu.de/sites/default/files/media/dokumente/05 1111 Koalitions vertrag Langfassung navigierbar 0.pdf, Zugriffsdatum: 10.07.2015

CDU/CSU und SPD (Koalitionsvertrag, 2013): Deutschlands Zukunft gestalten Koalitionsvertrag zwischen CDU, CSU und SPD, 2013, https://www.cdu.de/sites/default/files/media/dokumente/koalitionsvertrag.pdf , Zugriffsdatum: 03.08.2015

Devereux, M. P./Loretz, S. (National Tax Journal 2013): What Do We Know about Corporate Tax Competition?, in: National Tax Journal 2013, S. 745-773

DIHK (Realsteuervergleich, 2015): Bundesweite Übersicht der Realsteuer-Hebesätze 2015 in Städten über 20.000 Einwohnern, 2015, http://www.dihk.de/themenfelder/recht-steuern/steuern/finanz-undhaushaltspolitik/realsteuer-hebesaetze, Zugriffsdatum: 20.08.2015

Djanani, C./Grossmann, T. (StuW 2015): Kalte Progression aufgrund fehlender Inflationskorrektur außertariflicher Abzüge und Höchstbeträge bei der Einkommensteuer, in: StuW 2015, S. 33-51

DWS (Hrsg.) (Zukunft Erbschaftsteuer, 2015): Zukunft der Erbschaft- und Schenkungsteuer, Berlin 2015 
Eichfelder, S./Hechtner, F. (DStZ 2013): Belastungs- und Folgewirkungen der Anhebung des Grundfreibetrags in den Jahren 2013 und 2014, in: DStZ 2013, S. 227-242

Erkis, G. (DStR 2015): Der Entwurf zur Anpassung des ErbStG an das BVerfG-Urteil v. 17.12.2014 - "minimalinvasiv" oder "maximaladministrativ"?, in: DStR 2015, S. 1409-1413

Europäische Kommission (List of Prior Actions, 2015): List of Prior Actions, 2015, http://europa.eu/rapid/press-release_IP-15-5270_en.htm, Zugriffsdatum: 30.06.2015

Eurostat (Bruttoinlandsprodukt, 2015): Bruttoinlandsprodukt 2014, 2015, http://ec.europa.eu/eurostat/tgm/table.do?tab=table\&init=1\&plugin=1\&pcode=t ec00001\&language $=$ de, Zugriffsdatum: 23.07.2015

FG Hamburg, Beschluss vom 29.2.2012, 1 K 138/10, DStRE 2012, S. 478

Gemeindefinanzkommission - AG "Kommunalsteuern" (Abschlussbericht, 2011): Abschlussbericht, 2011

Grünwald, K./Friz, F. (DStR 2012): Verfassungsrechtliche Zweifelsfragen bei der Hinzurechnung von Miet- und Pachtzinsen nach $\S 8$ Nr. 1 Buchst. e GewStG bei gewerblichen Zwischenvermietern, in: DStR 2012, S. 2106-2111

Halaczinsky, R. (DStR-Beihefter 2014): 50 Jahre Einheitswerte 1964 - Bewertung des Grundbesitzes in den alten Bundesländern, in: DStR-Beihefter 2014, S. 139-148

Hannes, F. (ZEV 2015): Der Regierungsentwurf zur Reform der Unternehmenserbschaftsteuer, in: ZEV 2015, S. 371-377

Hechtner, F. (StuW 2014): Das Gesetz zum Abbau der kalten Progression: Baut die Anhebung des Grundfreibetrags die kalte Progression vollständig ab?, in: StuW 2014, S. 132-144

Heinemann, F./ Spengel, C./ Bräutigam, R./Evers, M.-T. (Quantitative Auswirkungen Erbschaftsteuer, 2015): Das Eckpunktepapier und der Referentenentwurf des BMF zur Erbschaftsteuer, 2015

Heuermann, B. (DStR 2013): Steuerinnovation im Wandel: Einige Thesen zur Zinsschranke und ihrer Verfassungsmäßigkeit, in: DStR 2013, S. 1-5

Hey, J. (DB 2015): Neustart durch temporäre Abschaffung der Erbschaftsteuer, in: DB 2015, S. Heft 26, S. M25

Houben, H./Maiterth, R. (Perspektiven der Wirtschaftspolitik 2010): Breite Bemessungsgrundlage und niedriger Proportionaltarif als alternative Erbschaftsteuerreform - Eine empirische Analyse, in: Perspektiven der Wirtschaftspolitik 2010, S. 204-222 
Jacobs, O. H./ Scheffler, W./ Spengel, C./ Bergner, S./ Bräutigam, R./ Keilen, B./Mayer, G. (Unternehmensbesteuerung, 2015): Unternehmensbesteuerung und Rechtsform: Handbuch zur Besteuerung deutscher Unternehmen, 5. Auflage, München 2015

Jacobs, O. H./Spengel, C. (European Tax Analyzer, 1996): European Tax Analyzer: EDVgestützter Vergleich der Steuerbelastung von Kapitalgesellschaften in Deutschland, Frankreich und Großbritannien, Schriftenreihe des ZEW, Band 11, Baden-Baden 1996

Keller, D. (Südwest Presse 06.08.2015): Grundsteuer: Eine mühsame Reform, in: Südwest Presse vom 06.08.2015, http://www.swp.de/ulm/nachrichten/wirtschaft/Grundsteuer-Eine-muehsameReform;art4325,3367490, Zugriffsdatum: 10.08.2015

Keß, T. (FR 2013): Keine ernstlichen Zweifel an der Verfassungsmäßigkeit der Hinzurechnungen nach $\S 8$ Nr. 1 GewStG 2002 n. F., in: FR 2013, S. 188-189

Kirchhof, G. (DStR 2015): Verfassungsrechtliche Grenzen der Erbschaftsteuerreform und eine auf die Leistungsfähigkeit ausgerichtete "Bedürfnisprüfung", in: DStR 2015, S. 1473-1481

Korezkij, L. (DStR 2015): Erbschaftsteuerreform: Änderungen durch den Regierungsentwurf vom 8.7.2015, in: DStR 2015, S. 1649-1653

Krumm, M. (FR 2015): Bewertung und Verschonung von sog. großen (Familien-) Unternehmen nach der Entscheidung des BVerfG v. 17.12.2014 zur Erbschaftsteuer, in: FR 2015, S. 481-496

Ministerium der Finanzen Brandenburg (Übersicht GrESt-Sätze, 2015): Grunderwerbsteuer - Hintergrundinformation, 2015, http://www.mdf.brandenburg.de/cms/detail.php/bb1.c.382984.de\#kap7 Zugriffsdatum: 13.08.2015

Nehls, D./Scheffler, W. (ifst-Schrift 503, 2015): Grundsteuerreform: Aufkommens- und Belastungswirkungen des Äquivalenz-, Kombinations- und Verkehrswertmodells, ifst-Schrift 503, Berlin 2015

Pahlke, A. (ZEV 2015): Reform der Erbschaftsteuer durch Abbau von Verschonungsregelungen?, in: ZEV 2015, S. 377-382

Prinz, U. (DB 2014): Zinsschranke vor dem Scheitern? Anmerkungen zum BFHBeschluss vom 18. 12. 2013 - I B 85/13 , DB 2014 S. 927, in: DB 2014, S. 11021103

Redoano, M. (European Journal of Political Economy 2014): Tax Competition among European countries Does the EU matter?, in: European Journal of Political Economy 2014, S. 353-371

Ritzer, C. (DStR 2013): Hinzurechnung von Finanzierungsanteilen nach $\S 8$ Nr. 1 GewStG, in: DStR 2013, S. 558-565 
Roth, H.-P. (SteuK 2015): Erbschaftsteuerreform: Änderungen im Regierungsentwurf vom 8.7.2015, in: SteuK 2015, S. 291-293

Scheffler, W. (DB 2015): Die Grundsteuer ist reformbedürftig, in: DB 2015, S. Heft 8, S. M5

Seer, R. (GmbHR 2015): Überprivilegierung des Unternehmensvermögens durch §§ 13a, 13b ErbStG - Zum Urteil des BVerfG vom 17.12.2014 - 1 BvL 21/12 -, in: GmbHR 2015, S. 113-121

SPD (Kalte Progression, 2014): Schluss mit der Kalten Progression, 2014, http://www.spd.de/aktuelles/122416/20140804 kalte progression.html, Zugriffsdatum: 01.07.2015

Spengel, C. (StBW 2012): Neuordnung der Grundsteuer - Anforderungen, Ziele und Gestaltungsmöglichkeiten, in: StBW 2012, S. 31-36

Spengel, C./ Bergner, S./ Bräutigam, R./ Evers, M.-T./ Hausemer, P./Plances, S. (SME Taxation in Europe, 2015): SME Taxation in Europe - An empirical study of applied corporate income taxation for SMEs compared to large enterprises, Brussels 2015

Spengel, C./ Bräutigam, R./Evers, M.-T. (DB 2014): Steuerbelastung von Kapitalgesellschaften in der EU, in: DB 2014, S. 1096-1101

Spengel, C./ Endres, D./ Finke, K./Heckemeyer, J. H. (Effective Tax Levels Using the Devereux/Griffith Methodology, 2014): Effective Tax Levels Using the Devereux/Griffith Methodology, Mannheim 2014

Spengel, C./ Heckemeyer, J. H./Zinn, B. (DB 2011): Reform der Grundsteuer: Ein Blick nach Europa, in: DB 2011, S. 10-14

Spengel, C./Nusser, H. (Der Konzern 2015): Aktueller Stand des OECD-BEPS-Projekts und mögliche Konsequenzen für die Konzernbesteuerung, in: Der Konzern 2015, S. $9-15$

Spengel, C./Zinn, B. (StuW 2011): Vermögensabgaben aus ökonomischer Sicht - Eine quantitative Analyse unter Berücksichtigung aktueller politischer Reformvorschläge, in: StuW 2011, S. 173-188

Watrin, C./Eberhardt, D. (IStR 2013): Besteuerung von Streubesitzdividenden und internationale Steuerplanung, in: IStR 2013, S. 814-820

Wissenschaftlicher Beirat beim BMF (Grundsteuerreform, 2010): Reform der Grundsteuer, 2010

Wissenschaftlicher Beirat beim BMF (Erbschaftsteuer, 2012): Die Begünstigung des Unternehmensvermögens in der Erbschaftsteuer, 2012 